\title{
The Relativistic Giant Atom: Evidences and Recommendations
}

\author{
Emad Eldieb \\ Forensic Sciences Organization, Cairo, Egypt \\ Email: eeldieb@yahoo.com
}

How to cite this paper: Eldieb, E. (2018) The Relativistic Giant Atom: Evidences and Recommendations. Open Access Library Journal, 5: e3797.

https://doi.org/10.4236/oalib.1103797

Received: July 2, 2017

Accepted: April 10, 2018

Published: April 13, 2018

Copyright $\odot 2018$ by author and Open Access Library Inc.

This work is licensed under the Creative Commons Attribution International License (CC BY 4.0).

http://creativecommons.org/licenses/by/4.0/

\begin{abstract}
In 2017 we published a new theory under the title: physics of the relativistic giant atom [1], where we suggested an alternative solution for the theory of special relativity. Some points were ambigious. Here in this present article we rewrite the same paper with clarification of the ambigios points, and with adding new ideas. If the original relativity says: the relativistic mass $m_{(v)}$ of a particle increases and its relativistic length decreases with a defined $\gamma$ factor hence, the hidden meaning of this original solution is appearance of the relativistic linear mass density in the form of $\frac{m}{r}=\left(\gamma m_{o}\right)\left(\frac{\gamma}{r_{o}}\right) \equiv \gamma^{2}\left(\frac{m_{o}}{r_{o}}\right)$. This
\end{abstract} rearrangement may allow-under defined conditions - an alternative relativistic solution in the form of creation of new particles counting $\gamma^{2}$, each with the same original rest mass $m_{o}$ and the same original rest radius $r_{o}$. This study searches in the conditions required for this alternative solution as follow: if we wrote the famous Einstein's equation of the rest mass of a particle (let it, a proton) as: $k \frac{e^{2}}{r_{o}}=E_{o}=m_{o} c^{2}$ then this form describes the particle electromagnetic energy in a rest state, where the proton doesn't feel-from interiorits charge and consequently this equation cannot describe repulsive electric energy. If we multiplied the left side of this equation by the number one then the result should be the same right side, and the equation still describes the proton in its rest state. Now if we defined a sphere $s$, with a quantity of protons $p$, distributed homogeneously among much more number of neutrons $n$, and if we have a quantity equal the number one as: $\left(\frac{r_{o}}{r_{c}} \frac{p^{\frac{2}{3}}}{\left(\frac{n}{p}\right)^{\frac{1}{3}}}\right)=(1) \quad$ (where; 
$r_{c}$ is the spacing between particles).

$\therefore k \frac{e^{2}}{r_{o}}\left(\frac{r_{o}}{r_{c}} \frac{p^{\frac{2}{3}}}{\left(\frac{n}{p}\right)^{\frac{1}{3}}}\right)=k \frac{e^{2}}{r_{o}}(1)=k \frac{p e^{2}}{r_{s}}=E \equiv E_{o} \equiv m_{o} c^{2}$

(where $r_{s}$ is the radius of the sphere while $c$ is the speed of the electromagnetic energy and not real particle speed). This nonrelativistic equation is still carrying the same meaning, and it is-still-describing the rest mass equivalent energy of the same particle with the same rest state defined by the new spacing $r_{c}$ (in addition to its inertial description defined by its rest radius $r_{o}$ ). The hidden meaning here is condensation of the particles in this new rest state. This last equation realizes two results: first, it describes the particle in its rest state, so the sphere can absorb physically all the repulsive \& exclusive energies, and second, it realizes the initial conditions: $m=m_{o} \& r=r_{o}$ (where the Coulomb's equation form is now fixed on the Einstein's form) hence, we may conclude an alternative relativistic solution in the form of: $n=\gamma^{2} n_{o}$. This idea led me to put a base for physics of self-replication as application of the relativistic giant charge which is ultra-cold fermions condensate. This state has the relativistic efficiency to make the particles replicate and withdraw all the kinetic energy. When we followed up physics of the giant atom we found complete coincidence between the estimated parameters of its orbit and the astronomic parameters of the solar system. This means that the solar system - in one of its primordial evolutionary stages-was a giant atom like system, and in the same time gives documents for the correctness of physics of the giant atom.

\section{Subject Areas}

Quantum Mechanics

\section{Keywords}

Quantum Physics, Relativistic Mass, Microscopic State, Ultra-Cold Neutrons, Solar System

\section{Introduction}

In this paper we put the relativistic basis for the giant atom like system [1]. Before we go with the discussion we have to stress on that it may appear in the beginning of this long study that there was contradiction with the current knowledge like the constant speed of light, Pauli's exclusion principle, and like quantum physics, but as soon as possible we would find that the study completely respects such facts. Also we have to remember all over this study that the final macroscopic state of the microscopic events inside the giant charge is the absolute zero energy except from a bit quantity of energy. We would divide the dis- 
cussion into two parts: the first would discuss physics of the relativistic giant charge, while the second will discuss the solar system in its primordial evolutionary stage as giant atoms, where we would discover that there is complete coincidence between the estimated parameters of the orbits of the giant atoms and that of the solar system as a document for the past existence of the giant atom system. The first part of this study discusses physics of the giant charge, while the second part discusses physics of this giant atom like system as orbital, and gives evidences for possibility of existence of such system.

Throughout the first part of this study we will find that the relativistic giant charge unit, like the giant proton unit (Compton sphere) having a quantity of elementary particles equal $n=10^{21}$ would produce-under a defined physical condition-the alternative relativistic solution in the form of replication by copy and paste to appear as a giant proton (the adult) having quantity of particles equal $N=10^{45}$. The careful mathematic manipulation with relativity would reveal that the relativistic particle mass (inside the giant charge unit) would not increase by its high speed (special relativity will not affect the mass of the elementary particle) but instead, the particles quantity is the one which increases (the substitutive relativistic solution would be in the form of increase the particles count from $n$ into $N$ ). This means that the energies of the electric and that of the relativistic gravitational field $u_{G}$ and also of the quantum state would be withdrawn giving finally a macroscopic rest state (the relativistic mass $m=$ the rest mass $m_{o}$ ).

Explicitly, the process of self-replication is the relativistic alternative solution. So if you looked for physics of this unit you would convince that the rest state is the macroscopic net state, or you can put the growth action in place of the motion action. This study stands on two physical bases. The first one is to put the coulomb's form of the electrostatic energy in the form of the famous Einstein's equation of the rest mass of a particle, and incorporate the reduced Compton's wavelength $r_{c}=r_{o}\left(\frac{r_{c}}{r_{o}}\right)$ as the spacing between particles where the rest radius of the particle $=r_{o}$.

To guarantee that we deal with real matter, and to guarantee that the quantum speed-which is arising from this spacing-is less than the speed of light we mathematically derived this spacing as just a bit above $r_{c}$ and consequently the quantum speed $v$ just a bit below $c$. The second idea is to use the effect of the special relativity on the gravitational and electric energy $u_{g} \& u_{e}$ and also on the quantum energy $u_{c}$ inside this sphere. To approximate this effect let us imagine a body has constant speed $v$, and on the same time it moves under the acceleration $a=\mathrm{d} v / \mathrm{d} t$, the relativistic gravitational force $=F_{G}=m a=m \frac{\mathrm{d}}{\mathrm{d} t} v / \sqrt{1-\beta^{2}}=\gamma^{3} m a$ while the same body with the same $v$ would hold relativistic electromotive force $F_{e}=k e^{2}\left(\gamma^{2} / r^{2}\right)$. Where, the charge $e$, is invariant. So, although the nonrelativistic $F_{e}$ is much bigger than the nonrelativistic $F_{g}$ yet the relativistic $F_{G}$ may get equal to the relativistic electric energy. If such equality happened, suspense 
would occur.

The study would be clear step by step. The Equations (56)-(68) would clarify the ambiguous points. I advise the reader to record his objections till he reaches to Equation (68). The author stresses on that, we have to be brave and accept the correct mathematic results whatever it may have suspense. The goal is to put the electric repulsive energy-per a proton-inside this sphere, in the Einstein's rest form. Once this occurs then we get two results. The first is; we have to search for alternative relativistic solution (other than the relativistic mass \& length). The second is; we succeeded, macroscopically, to abort-physically-the electric repulsive energy. We-also-can find the equations packet which would abort physically the quantum exclusive energy due to the spacing between the particles.

\section{The Compton's Sphere in a Rest State}

From the relativistic relation $m^{2}=m_{o}^{2} / 1-\beta^{2}$ where $\beta=v / c$, if the speed $v$ of a particle $=c$ then the relativistic mass $m$ would be infinite (refused), and if $v^{2}=2 c^{2}$ then, $m^{2}=-m_{o}^{2} \quad$ (also is refused).

The Einstein's famous equation of the rest mass [2] of a subatomic particle like a proton (or neutron...) is defined by

$$
k \frac{e^{2}}{r_{o}}=m_{o} c^{2}
$$

where, it is positive energy equating the relation between the inertial rest mass $m_{o}$ of the particle and its rest mass equivalent energy $E_{o}$ (where $k$ stand for the electrostatic constant $=8.98 \times 10^{9} \mathrm{~N} \cdot \mathrm{m}^{2} / \mathrm{C}, e$ is the value of the elementary charge in coulomb and $r_{o}$ is the classic rest radius of the particle). If we multiplied the left side by the number 1 then the right side should be typically the same. On the same form, if we defined a system (neglect quantum mechanics for a moment) formed of quantity of protons $p$, distributed homogeneously among much more number of neutrons $n$, such that $\frac{n+p}{p} \approx n / p$ and if we defined the quantity $\left(\frac{r_{o}}{r_{c}} \frac{p^{\frac{2}{3}}}{\left(\frac{n}{p}\right)^{\frac{1}{3}}}\right)=1$

$$
\therefore k \frac{e^{2}}{r_{o}}\left(\frac{r_{o}}{r_{c}} \frac{p^{\frac{2}{3}}}{\left(\frac{n}{p}\right)^{\frac{1}{3}}}\right) \equiv m_{o} c^{2}
$$

where, the relativistic mass here - in this equation $-m=$ the rest mass $m_{o}+0=$ $m_{o}$ and consequently $r=r_{o}$ (If-and only if-the product inside the big brack- 
ets $=1$ and consequently, if $v=c=$ speed of the electromagnetic energy e m.e). Let us call this system "Compton's sphere". Now let us-also-multiply the electric field contributor of $e$ m.e of the rest particle by the number one so let us define:

$$
\frac{1}{2} k \frac{e^{2}}{r_{o}} \times 1=\frac{1}{2} m_{o} c^{2} \times 1
$$

Because of we can define any quantity of $p$ and $n$ so let us-also-define another quantity as:

$$
\left(\frac{r_{o}}{r_{c}} \frac{p^{\frac{2}{3}}}{\left(\frac{n}{p}\right)^{\frac{1}{3}}}\right)=\frac{1}{2}
$$

Now again, let us-for a moment-neglect the relativistic \& quantum effect and let us imagine that we have so big quantity of protons inside this Compton's sphere that the repulsive electric energy takes the form of:

$$
k \frac{e^{2}}{r_{o}}\left(\frac{r_{o}}{r_{c}} \frac{p^{\frac{2}{3}}}{\left(\frac{n}{p}\right)^{\frac{1}{3}}}\right) \equiv \frac{1}{2} m_{o} c^{2}
$$

We can see this equation as a physical description for the electric potential of the electromagnetic energy of a particle in a rest state (see Appendix) and-also-we can see it as a physical description for the repulsive electric energy for each particle. But we have to notice that: the original relativistic solution describes the kinetic energy $u_{e}=\gamma m_{o} c^{2}-\Delta m c^{2}$ while our alternative solution begins from the idea of Equation (2) which describes the particle (definitely, inside our sphere) by non-relativistic mass (see later), so, we can formulate:

$$
u_{e}=m_{o} \int v \mathrm{~d} v=\frac{1}{2} m_{o} v^{2}
$$

The important point-here-is to notice that the number one is not arbitrary mathematic number because it carries a physical meaning, so it could not-here-replaced by any other number. The number one when be multiplied by Equation (1) gives the physical meaning of the energy of a particle in a rest state, and when be multiplied by Equation (3) it gives the physical meaning of the electric potential subdivision of this electromagnetic energy (half of the stored energy) of the particle in the same state. This means that multiplication by other numbers to get the same result is physically wrong. For example, we cannot multiply the left side of the first equation say, by 0.1 to get in the other side rest particle with $0.1 m_{o} c^{2}$ (mathematics sometimes have to think as a physician). Also we have to notice that the two sides of each of Equation (3) \& Equation (5) are synonyms, so to conserve this relation we have to do first; an 
added deficit quantity in one side have to be compensated by an added equal excess quantity in the same side, and second the same operations have to be hold on the other side of the equation. Also we have to notice that the equations which describe half of the rest particle are physically not independent equations because finally we have to describe a complete particle. So, such equations should be considered as a temporary station just to describe the micro events of the macro-state. Hence, we can do a junction between Einstein's equation form, which talks about a particle in a "rest state" and coulomb's equation form which talks finally about "relativistic mass". Since, this coulomb's form takes the same form of Einstein's equation (if we neglected-for a moment-the original relativistic relations), so it is acceptable to carry the two meanings (rest mass \& relativistic mass). When the two meanings are present in the same equation, then this may be explained by presence of "alternative" relativistic solution which affects something other than the rest mass. But the thing which acts against this coulomb's form is that, it carries the meaning of real speed $v=c$ which is-from the side of view of relativity theory-refused. So let us define infinitesimal small quantity with minus sign (deficit) \& with positive sign (excess) and let this zero quantity appears in Equation (5) as follow:

$$
\left(k \frac{e^{2}}{r_{o}}\left(\frac{1}{2}\right)-\frac{1}{2} k \frac{e^{2}}{r_{o}} \frac{n}{p \mu}\right)+\frac{1}{2} k \frac{e^{2}}{r_{o}} \frac{n}{p \mu}=\left(\frac{1}{2} m_{s} c^{2}\right)+\frac{1}{2} k \frac{e^{2}}{r_{o}} \frac{n}{p \mu} \equiv \frac{1}{2} m_{o} c^{2}
$$

where $\frac{n}{p}$ is the ratio between the two unknowns $n$ and $p$ while $\mu$ is another unknown, all should be defined. Since, we want to study physics of collection of particles in this Compton's sphere which has the highest possible exclusive (the electric \& quantum) acquired energy, then, the particle never get energy more than that defined in Equation (6) and so, this equation could be considered the key for studying this case. But because $v=c$ is forbidden by the special relativity, so we need an infinitesimal cutoff, therefore, Equation (6) appears with the deficit only as:

$$
k \frac{e^{2}}{r_{o}}\left(\frac{r_{o}}{r_{c}} \frac{p^{\frac{2}{3}}}{\left(\frac{n}{p}\right)^{\frac{1}{3}}}-\frac{1}{2} \frac{n}{p x \mu}\right)=\frac{1}{2} m_{o}(\sim c)^{2}
$$

This coulomb's form (kindly, look at the integral form of Equations (41) \& (42)) talks about a sphere which neglects each of the relativistic relations \& the quantum effect and so it respects only the relativistic rule that $v<c$.

But actually, this acquired speed is a microscopic picture, while the final form of our sphere obeys Equation (3) or Equation (2) so its macroscopic state is the rest state. This means that $c^{2}$ which appears in (3) does not describe acquired speed but it just to describe the electric potential subdivision of the rest mass. So, $v=c$ is the speed of the electromagnetic energy (not real speed), hence, it is not refused in this sphere (where $m_{o} c^{2}$ means particle in a rest state). Therefore, 
the problem-here-which our system avoids is not $v=c$ but it is $v>c$ (if we neglected, the relativistic relations, hence the distance intervals in the primed and non-primed frames are equal: $\mathrm{d} x=\overline{\mathrm{d} x}$ therefore, the approaching frames can add their velocities). Fortunately, our alternative relativistic solution prevents $v>c$ but by the dynamics of Equation (2) (where the particle appears in the rest state $m_{o} c^{2}$ ) or Equation (6) where the particle appears in the rest state $m_{s} c^{2}$.

This problem arises from that; since the gravitational energy $\left(u_{g}\right)$ never waste from matter then it would add energy to the energetic function of the system (this ambiguous sentence would be clear in the next sections) which means excess speed over $c$. Hence, the deficit quantity which appears in the left side of (7) aims to avoid this problem. This is good news, because this means that the deficit in energy equals $u_{g}$ which would help us to define the unknowns. Equation (6) carries the same description of Equation (5) and could be considered its microscopic shape, while (7) was formulated to show and concentrate on this microscopic action. It is clear from the deficit inside Equation (7) that it initiates the relativistic action. But each of the two equations in the above form is still describing the rest mass (no relativistic mass appears) so this form may carry the meaning of the alternative relativistic solution. Since the equation does not speak about external energy (it just describes the potential or its rest energy), so its inertial rest $c^{2}$ with that cutoff (deficit) appears as real speed. This speed would launch the relativistic action which appears if-and only if-we began from (7). This action-as we will see-would add first; excess quantity equal the deficit, so we go back to Equation (6). And second, it would add the quantity D which is defined by Equation (9) (see next) so we go back to Equation (2) or Equation (1) which is just describing a particle in a rest state which means that the relativistic mass is the same the rest mass. Hence, we are in an urgent need to the alternative relativistic solution. Finally, we would discover that the entire equations of this sphere are not more than description of the energy of a particle in a rest state. Equation (2) could be written mathematically in the form of Equation (6) as:

$$
\left\{\frac{1}{2}\left(k \frac{e^{2}}{r_{o}}-\frac{k \frac{e^{2}}{r_{o}} n}{p x \mu}+\frac{k \frac{e^{2}}{r_{o}} n}{p \times \mu}\right)\right\}+\left\{\frac{1}{2}\left(k \frac{e^{2}}{r_{o}}-\frac{k \frac{e^{2}}{r_{o}} n}{p x \mu}+\frac{k \frac{e^{2}}{r_{o}} n}{p \times \mu}\right)\right\} \equiv \frac{1}{2} m_{o} c^{2}+\frac{1}{2} m_{o} c^{2}(8)
$$

Let the second term $=D$, the excess quantity $=b \&$ the deficit $=d$

$$
m_{o} c^{2} \equiv\left(\frac{1}{2} k \frac{e^{2}}{r_{o}}-\frac{1}{2} \frac{k \frac{e^{2}}{r_{o}} n}{p x \mu}\right)+b+D=(a+b)+D=(f-d)+b+D
$$

If we delayed the definition of the spacing $r_{c}$ to a next chapter, then Equation (7) could be considered as a junction between Einstein's \& coulomb's equa- 
tion forms, from which we can declare launching physics of the giant atom. In the next chapter we would put in consideration the relativistic effect and then in a following chapter we would consider the quantum \& uncertainty effect.

\section{The Rest Particle inside a Collection}

To conserve the initial conditions $\left(m=m_{o} \& r=r_{o}\right)$ we need to fix the coulomb's form on the form; $k \frac{e^{2}}{r_{o}}=m_{o} c^{2}$ where the initial conditions are implied in this Einstein's form. Since, the factor $c^{2}$ does not imply real speed so this form of equation means abortion of the electric repulsive energy of the coulomb's form inside the Compton's sphere. All the micro-events which would appear in this study should be directed physically to realize-finally-the initial condition of the macro-state of Equation (1), that is to say, we will choose-all over the study - the solution which realizes the rest state of the particle inside this sphere. It is the fatal mistake if we forgot this idea. If we want to talk about real speed and to avoid real speed $v=c$ and-also-to initiate the action of physics of the sphere let the speed $v$ of the sphere particles be close to $c$ therefore, let us define $v \sim C$ where the deficit would appear as:

$$
v^{2} \approx c^{2} \rightarrow v^{2}=c^{2}\left(1-\frac{n}{p x \mu}\right)=c^{2}\left(1-\frac{1}{\gamma^{2}}\right)=c^{2}\left(1-\frac{1}{c_{o}}\right)
$$

This form talks about real speed so the electrostatic energy is so great that the relativistic relations are considerable.

Let us rewrite (6) as:

$$
\left\{\frac{1}{2} m_{o} c^{2}\left(1-\frac{n}{p x \mu}\right)\right\}+\frac{1}{2} m_{o} c^{2} \frac{n}{p x \mu}=\left\{\frac{1}{2} k \frac{e^{2}}{r_{o}}-\frac{1}{2} \frac{k \frac{e^{2}}{r_{o}} n}{p x \mu}\right\}+\frac{1}{2} \frac{k \frac{e^{2}}{r_{o}} n}{p x \mu}
$$

And let us in this chapter consider only the contents of the outer brackets of the two sides which are equivalent to (7). The goal here is to see how the relativistic action-due to the deficit-produces the " $D$ " term and also to see the urgent physical need for the alternative relativistic solution. Since the left side of Equation (1) or Equation (3) is a synonym to the right side, so adding a deficit and an excess quantity to the left side should appear-also-in the right side as in Equation (11). Actually (7) is the firing equation of the Compton's sphere. But since the final macroscopic state would appear in the complete form of Equation (11) or Equation (1) so we will see that the Compton's sphere is in complete rest state; the deficit quantity was a deficit in the rest mass of the left side of the equation and necessarily, a deficit of the rest energy of the right side, while the compensating excess term is the nonrelativistic gravitational energy which equals the equivalent energy of that deficit. This deficit in the equation is the first block in physics of the giant charge which will enable us to visualize the 
microstates of this equation. Now let us use the relativistic relations, so let us put

$$
\beta^{2}=\frac{v^{2}}{c^{2}} \text { And let, } \gamma^{2}=c_{o}=\frac{1}{1-\beta^{2}}
$$

where; $c_{o}=\varphi(v)$. Let us-also-define another unknown as; $\mu=\varphi(v)$,

$$
\gamma^{2}=c_{o}=\frac{1}{1-\beta^{2}}=\mu \frac{p}{n}
$$

where $\frac{n}{p}$ is constant.

$$
\therefore m^{2}=\gamma^{2} m_{o}^{2}=c_{o} m_{o}^{2}=\mu \frac{p}{n} m_{o}^{2}
$$

This is the original relativistic solution with the expression of these unknowns. Of course, one of the goals is to derive the value of this speed $v$ (\& density of the matter of this sphere) and consequently the values of these unknowns. The physical meaning of the last four equations appears from the next section.

\section{Global View}

1) Put the free particle described by Equation (1) inside the collection described by Equation (5). This gives a mathematic solution for the collection, but does not give the physical solution. According to the next Equation (14) and Equation (15), we can see relativistic gravitational potential $u_{g}(\gamma)=u_{G} \gg u_{e}(\gamma)$, and hence, the sphere would reach to equilibrium in a certain point. This idea can be the starting point of the physical solution. Now, we can define extreme electric initial speed as Equation (10), and also, we can define nonrelativistic gravitational potential:

$$
u_{g}=\Delta m c^{2}=m_{o} \frac{c^{2}}{c_{o}}
$$

And from Equation (12) we can get: $u_{g}=\Delta m c^{2}=m_{o} \frac{c^{2}}{c_{o}}=m_{o} c^{2} \frac{n}{p x \mu}$.

Hence, we understand that we can control and define the arbitrary quantities inside the sphere such that finally we get

$$
u_{\mathrm{g}}=\Delta m c^{2}=m_{o} \frac{c^{2}}{c_{o}}=m_{o} c^{2} \frac{n}{p x \mu}=\frac{k \frac{e^{2}}{r_{o}} n}{p x \mu}
$$

And hence, we can understand the physical meaning of Equations (6)-(11).

2) Equations (20)-(27), can discover the mathematics of how each of $u_{G}$ and $u_{e}$ looks as:

$$
\begin{aligned}
& \frac{1}{2} m_{o}\left(c^{2}-\frac{c^{2}}{c_{o}}\right), \text { and how they add: } \\
& \qquad u_{G}+u_{e}=u \equiv m_{o}\left(c^{2}-\frac{c^{2}}{c_{o}}\right)
\end{aligned}
$$

3) This sphere represents a bound system. The bound system has a lower 
potential energy than the sum of its constituent parts. Its mass must be less than the total mass of its unbound constituents. In a separate section we would define the potential energy which binds the constituents of the sphere. If we looked to this sphere as a large nucleus, then all the energies-of the semi-empirical mass model-vanish, except the asymmetry and the Coulomb terms, in addition to the new term which is extremely considerable here in this very great nucleus. We would see how both, the electric repulsive and the quantum exclusive energies are aborted inside this sphere. The future-as we will see soon-carries something bad for the compound: $u_{G}+u_{e}$. This $u_{G}$ will represents something other than central binding energy. Hence, the system as a bound state should create a free $u_{g}$. This means that the particle mass should lose a little bit.

Since, the rest-particle mass after missing $\Delta m$ becomes $m_{s}\left(\right.$ not $m_{o}$ )

$$
\left(m_{o}-\Delta m\right)=m_{s}=\left(m_{o}-\frac{m_{o}}{c_{o}}\right)=m_{o}\left(1-\frac{1}{c_{o}}\right)
$$

By the law of conservation of energy, the energy of the system remains a constant $(u=$ constant $)$ :

$$
\therefore v^{2}=c^{2}\left(1-\frac{1}{c_{o}}\right) \div\left(1-\frac{1}{c_{o}}\right)=c^{2}
$$

$\therefore u=\left(m_{s} c^{2}\right)_{t}$. This is the intrinsic property of this magic sphere!!!

The cardinal idea is that: The factor that acts upon $u$ to conserve it, is the same factor which converts $v^{2}$ into $c^{2}$ or, the deficit in the mass due to the gravitational binding energy $\equiv$ the excess in $v^{2} \rightarrow c^{2}$

Hence, we can recall equation 2 to understand what the information which it carries. It say's: if $v^{2}=c^{2}$ like $\left(m_{s} c^{2}\right)$ then, it is-like Equation (1)-zero kinetic energy, such that, the energy which appears is rest energy (zero speed-state). So, the sphere succeeded to abort $u$ : the original relativistic relations vanish, and replaced by alternative relativistic relations defined by Equation (22) and (56).

In above, we labeled the rest energy of the system by the subscript $t$, to distinguish from the inertial energy of the rest particle. The next diagram summarizes the entire above. The rest particle would be labeled by the brackets \{\} while the energy of the system would be labeled by the brackets [ ] so no further need to the subscript $t$.

$$
\begin{gathered}
\left\{c^{2}\left[m_{o}\right] c^{2}\left(1-\frac{1}{c_{o}}\right)\right\} \\
\left\{m_{o} c^{2}\right\} \rightarrow\left\{m_{s} c^{2}\right\}+u_{g} \downarrow
\end{gathered}
$$

By the dynamics of conservation of $u$ :

$$
\left\{c^{2}\left[m_{s}\right] c^{2}\right\}+u_{g}
$$

It must be clear that the term $b$-which appeared in Equation (9) and which 
appears in other positions of the text-acts on $u$ by the above dynamics (by conservation of energy) not by else. Hence, we can again recall Equations (6)-(9) to understand how the term $b=u_{g}$ acts with the conserved term $u$

$$
\left\{k \frac{e^{2}}{r_{o}}-\frac{k \frac{e^{2}}{r_{o}} n}{p x \mu}\right\}+\frac{k \frac{e^{2}}{r_{o}} n}{p x \mu}=\left(k \frac{e^{2}}{r_{o}}-u_{g}\right)+u_{g}=m_{s} c^{2}+u_{g} \equiv m_{o} c^{2}
$$

Appearance of the term $m_{s} c^{2}$ is enough to convince that Equation (2) is realized and $u$ is aborted (it was transformed into rest energy). The two terms $m_{s} c^{2}+u_{g}$ mean that we have got the particle which is defined by Equation (1), but inside a collection. If you want the same free particle-defined by Equation (1) - to appear here, then we can add the sign of equivalency. The confused point here is that, if the relativistic $u_{G}$ had been aborted with its conjugate $u_{e}$, hence we can ask about how a new $u_{g}$ could be precipitated in the system. The answer you can find it in Section 9.

It is clear that the quantity $m_{s} c^{2}$ which appears always with the summation $\left(u_{G}+u_{e}\right)$ it is not-at all-the same original rest energy, although, it describes the original rest mass. Therefore, we have to distinguish between the inertial (internal) rest energy of the same original rest particle and the rest energy of the system which represents the subject of this article. Where did the kinetic energy go? Here is the answer:

We have two functions: one describes a constant real speed, while the second describes zero speed-state. Both should appear in a general solution. According to the original relativity, the kinetic energy appears as $m_{(v)} \varphi(v)=m_{o} \oint_{o}^{v} \gamma v \mathrm{~d} v$, while it appears in the alternative solution in the form: $m_{o} \varphi(v)=m_{o}\left(\frac{0+v^{2}}{2}\right)=\frac{1}{2} m_{o} v^{2}$. As you see, in this alternative solution, each of the mass and the speed is a constant and is neither a continuous nor a differential function. Hence, we can conclude: the general solution-which adds the two solutions-, represents a particle bouncing forth and backward-inside its defined space-between two points each with infinite potential. We need a discontinuous function to represent a harmonic but not simple motion (not sinusoidal):

$$
\begin{aligned}
\dot{x}(t) & =1 v:\left[0<t<\frac{T}{2}\right] \\
& =-1 v:\left[T>t>\frac{T}{2}\right] \\
& =c: t=\left\{\frac{T}{2}, T\right\}
\end{aligned}
$$

Such that $c$ represents the zero state (rest energy), while $v=c \sqrt{\left(1-\frac{1}{c_{o}}\right)}$.

The upper case letter describes the time of the oscillatory motion $T=\frac{r_{c}}{v}$. 
The last solution carries the meaning of $v^{2}=c^{2}$ which first, can-by the addition to the original rest energy of the particle $\left(m_{o} c^{2}\right)$-realize (as we will see) the general solution $v^{2}=2 c^{2}$ and second, could be considered as the macroscopic state or the boundary condition which should strictly define the first two microscopic sates. Hence, we have: $S=\gamma^{2} S$ (please look at Section 15 to realize that the relativistic particles number is a temporary stage, and we will return to the initial particles number: a fact which agrees with the familiar knowledge)

$$
\bar{m}=m, \bar{x}=x
$$

4) From all above, we can summarize: the particle inside this magic sphere appears as a "paper" formed of two faces. The particle itself looks as a point while the remainder of the surrounding is the energy of the system. On a face of the paper is written:

Special relativity-here-and only here, is constrained by Equation (1), therefore; the repulsive and the exclusive energies are aborted and consequently, the original relativistic relations are-also-aborted. This means constant density of matter.

While on the second face is written:

$$
\begin{gathered}
u_{e}=\frac{1}{2} m_{o} c^{2}\left(1-\frac{1}{c_{o}}\right) \\
u_{G}=\frac{1}{2} m_{o} c^{2}\left(1-\frac{1}{c_{o}}\right) \\
u=u_{e}+u_{G}=m_{o} c^{2}\left(1-\frac{1}{c_{o}}\right)
\end{gathered}
$$

Now, let us divide the sphere into two sub-spheres.

\section{The Relativistic Protons Potential Well \& the Approximate Solution}

Now, let a defined quantity of protons in a defined density of nucleons to allow each proton to gain the above defined speed $v \approx C$ (due to the electrostatic action) and let-also-the density of the same sphere allows each neutron to gain the same $v \approx C$ as quantum speed (due to the relative spacing $r \approx r_{c}$ ), then the relativistic gravitational energy as speed function $u_{G}(v)$ would be affected by the speed $v$ more than the electric energy $u_{e}(v)$ such that the factor $\frac{1}{\gamma}=\sqrt{\frac{1}{c_{o}}}=\left[1-\left(\frac{v^{2}}{c^{2}}\right)\right]^{1 / 2}$ appear in the denominator of the relativistic gravitational potential energy per a proton $u_{G}(v)$ with power $3 / 2$ while it appears in the denominator of the electric potential energy per a proton $u_{e}(v)$ only with power $1 / 2$

$$
\begin{gathered}
u_{G}=-G n\left(m_{o}^{2} c_{o}\right)\left(c_{o}^{1 / 2} / r_{s}\right) \\
u_{e}=k p e^{2} c_{o}^{1 / 2} / r_{s}
\end{gathered}
$$


where; $r_{s}$ is the radius of the sphere \& $G$ is the universal gravitational constant. As we saw in the beginning of this section-and to realize Equations (2) \& (5) - (we would see later that the magnetic moment energy of the sphere is close to zero, therefore $u_{G}$ would replace it to realize the intuitive mathematic solution) there should be a form of equality (that is to say, we are searching for the conditions required for this equality) appears in the form:

$$
-G n\left(m_{o}^{2} c_{o}\right)\left(c_{o}^{1 / 2} / r_{s}\right)=k \frac{p e^{2} c_{o}^{1 / 2}}{r_{s}}
$$

The right side of this equation represents the electric energetic function which should appear-in the Compton's sphere-in a rest form (one half of a particle in a rest state) while the left side represents the second half of the rest particle (see Appendix).

From the Einstein's equation form:

$m=m_{o} \& r=r_{o}$ is realized only at;

$$
m=m_{o} \& r=r_{o}
$$

is realized only at $k \frac{e^{2}}{r_{o}}=m_{o} c^{2}$. Because we began from the outer brackets of (11) — which carries the relativistic meaning — and not perfectly from (1) so we would consider (17) is a proposal realized only at existence of the terms $b$ \& $D$ defined in (9), and hence we would deal with the next equations on this base where later on we will study how the excess term is deposited in the equation. Let us now see how the term " $D$ " is added.

Hence, -on this proposal-we can do algebraic simplifications (abbreviations on the factor $c_{o}$ ) where finally the equality appears after simplification as:

$$
k \frac{e^{2}}{r_{o}}\left(\frac{r_{o}}{r_{c}} \frac{p^{\frac{2}{3}}}{\left(\frac{n}{p}\right)^{\frac{1}{3}}}\right)=\left(-G c_{o} \frac{m_{o}^{2}}{r_{o}}\right)\left(\frac{r_{o}}{r_{c}}\right) n^{2 / 3}
$$

where; $c_{o}$ is the equality factor (defined from Equation (12)) and acts in this equality by a vague process would be determined latter and the negative sign means that the two sides act opposite to each other. The solution in the form of asymmetric addition is;

$$
A+(-A)=0
$$

Let us search for other solution and so go back again.

Let us recall the equality; $k \frac{e^{2}}{r_{o}}\left(\frac{1}{2}\right)=-G c_{o} m_{o}^{2} \frac{n}{r_{s}}$.

Put $\left(-m_{o}^{2}\right)$ as a relativistic equivalent to $m^{2}$ (proposal should be realized). Although this is original (as we will see) but imaginary solution yet we would choose it because it is the one which realizes Equation (1) (as we will see soon).

$\therefore m^{2}=\left(-m_{o}^{2}\right)$ is a second proposal should be realized (20) 
Rewrite (18) in its simplified relativistic form

$$
\therefore G\left(-\gamma^{2} m_{o}^{2}\right) \frac{n}{r_{s}}=k \frac{e^{2}}{r_{o}}\left[\frac{r_{o}}{r_{c}} \cdot \frac{p}{(n)^{1 / 3}}\right]
$$

By substitution of (12) \& (20) in the left side and of (4) in the right side of (21)

$$
\therefore \frac{G m^{2}\left(n c_{o}\right)}{r_{s}}=k \frac{e^{2}}{r_{o}}\left(\frac{1}{2}\right)
$$

Since, $m=m_{o}$ therefore $c_{o}$ has physical concept which must appear as:

$$
\frac{G m^{2}\left(n c_{o}\right)}{r_{s}}=k \frac{e^{2}}{r_{o}}\left(\frac{1}{2}\right) \equiv \frac{1}{2} m_{o} c^{2}
$$

Since, $n$ is the counting of the neutrons, but we want the actual counting of the nucleons (where $n \gg p$ ) and so putting the actual count of the nucleons gives:

$$
\frac{\left.G m^{2}\left[(n+p) c_{o}\right)\right]}{r_{s}}=k \frac{e^{2}}{r_{o}}\left(\frac{1}{2}\right) \equiv \frac{1}{2} m_{o} c^{2}
$$

The middle side of this equation-like the left-has become positive energy; such that each side is-now-possessing the same magnitude and the same positive form of $\frac{1}{2} k \frac{e^{2}}{r_{o}}$ therefore the equality has-now-symmetric additive solution as:

$$
u=u_{e}+u_{G}=\frac{1}{2} m_{o} c^{2}+\frac{1}{2} m_{o} c^{2}=m_{o} c^{2}
$$

The term $D$ is-now-deposited in the additive form where each term of the left side represents the value of the energy of $u_{e} \& u_{G}$.

This equation does not describe a new created particle but it describes the Compton state energy (per a proton) - definitely_in a rest state, as equivalent to the right side of the equation, so it is a new description (originating from the Compton state) of the rest particle which when be added to its inertial description we get:

$$
m_{o} c^{2}+m_{o} c^{2}=m_{o} 2 c^{2}
$$

Equation (25) describes the total rest states $(0+0)$. So the proposed equality (20) has a solution appears simply from (25) and also has a relativistic concept in the form of:

$$
m^{2}=\frac{m_{o}^{2}}{1-\beta^{2}}=\frac{m_{o}^{2}}{1-2}=-m_{o}^{2}
$$

And from (26) it has an original relativistic solution as;

$$
v^{2}=2 c^{2}
$$

Equation (23) is the simplest form regarding the factor $c_{o}$ there for it is the correct form which shows the correct relativistic alternative solution. Equations (23)-(27) act as one packet. Equations (27) \& (25) are synonyms where they represent the condition required for existence of the giant charge where such 
state does not mean $v>c$ but it rather says that the particle is in a rest state but this state is defined by its Compton state as it is defined by its intrinsic properties $(0+0)$, or it definitely means relativistic solution for the proposal: $m^{2}=-m_{o}^{2}$ where the negative sign of $-m^{2}$ does not mean direction but it is an operator has a radical physical meaning such that it converted the kinetic electric $\&$ the relativistic gravitational energies in the equality of Equation (22) into conjugate pair (this expression means-all over the study-Equation (24)) of rest energy (the next section of this study will give further clear meaning for this imaginary factor). Anyway, the second proposal is now realized but if-and only if-Equation (27) was realized or definitely, if the term $b$ was deposited in the equation. The same equality of (20) when takes place in (18) - after understanding the physical meaning of its negative sign-leaves behind, the conclusion:

$$
m^{2}=-m_{o}^{2} \rightarrow m^{2}=m_{o}^{2} \rightarrow m=m_{o} \& r=r_{o}
$$

But this conclusion appears only inside (22) and the equations derived from it. So Equation (23) - which is the simplest form and which engulfed physically the negative sign of the proposal of Equation (20) - is the one which realizes excellently the initial condition which is discovered by Equation (24), so it is the one which carries the alternative relativistic solution. Implicitly, when Equation (20) takes place in (18) and when the symmetric addition takes place in (24) which carries the meaning of $m=m_{o}$ then, we are in urgent need to alternative relativistic solution and-also-we are still in the correct extension of Equation (1). But since our equations are built on the hidden deficit of Equation (11) where we saw only its relativistic action arising from $v \sim c$ or arising from the speed defined by Equation (10) so we would consider the obtained results need the correcting term $b$ appears with (7) to compensate that deficit. Till we discover this missed term we will consider the result of Equation (24) as an approximate solution. This correcting term and the dealing with Equation (7) not (6) (with $v \approx C$ not $v=c$ ) would appear in a separate chapter titled the deficit \& excess. Now let us go on with the alternative relativistic solution.

Equations (20) \& (27) realize each other in the special relativity and represent the second block in the giant charge physics.

Now from Equation (23) we can write the original relativistic mass in the form of:

$$
\left[\left(n_{o}+p_{o}\right) c_{o}\right] m_{o}^{2} \equiv(n+p) m_{o}^{2} \quad \text { where } m=m_{o}
$$

A new terminology can correct any misunderstand. So we can put the reproductive energy or the rest energy of the system in place of the kinetic energy. Dividing the two sides of Equation (22) over $p$ discovers the factor $\mu$ which appears (independently from Equation (12)) as:

$$
\mu=c_{o}\left(\frac{n}{p}\right)=\frac{\left(c_{o} n\right)}{p}=\frac{N}{p}
$$

Since; $\frac{n}{p}=$ constant 


$$
\therefore c_{o}=\gamma^{2}=N / n=P / p
$$

where; the capital letters $N$ and $P$ are the "relativistic" number of the neutrons and protons of the giant proton (the adult) while the small letters $n$ and $p$ are the "rest" number of the neutrons and protons of the giant proton unit (the zygote-like unit). Actually, the relativistic dilatation of the particle number is not strange in physics: as the velocity of an observer relative to an emissive surface increases the number of photons (due to time dilatation) encountered by the observer per unit area per unit time (intensity).

\section{Physics of Compton's Sphere}

1) Equation (2) describes the particle in a rest state by the energetic functions of the Compton's sphere (in addition to its inertial energetic functions) so the final result is a rest particle defined by the intrinsic properties of Compton's sphere (the new rest spacing $r_{c}$ ) in addition to its rest radius $r_{o}$.

2) Since the rest particle is defined by its electric \& magnetic fields, and since the superposition of the charges of the particles is the origin of the charges of the collection, so the sphere (collection) has electric flux:

$$
\varnothing=\oiint E \cdot \boldsymbol{n} \mathrm{d} s=\nabla \cdot E \iiint \mathrm{d} V=\frac{Q}{\varepsilon}
$$

where $\boldsymbol{n}$ (in this \& the next equation) is the unit vector in the outward direction of the sphere. And has magnetic flux:

$$
=\oiint B \cdot \boldsymbol{n} \mathrm{d} s=\nabla \cdot B \iiint \mathrm{d} V=0 \rightarrow \nabla \cdot B=0
$$

This means no magnetic charges inside the sphere, so we need a flux to replace that zero flux. Hence, the flux of the conservative gravitational energy $u_{g}$ can perform this role. But since the particles are oscillatory moving so this $u_{g}$ would appear as $u_{G}$ (you never forget that Compton's sphere energy is a copy from Equation (1), so its entire equations should go straight forward with this equation.

The goal in this point was to define so great speed that $u_{G}$ can represent the half of the energy of a rest particle. This was done by choosing defined number of particles within defined density (as we will see in the next sections).

3) Since, we need to realize Equation (2) (the initial conditions) so, we have to do algebraic simplifications on $\left(c_{o}\right)$ of $\left(m_{o} \& r_{o}\right)$, hence, we got Equation (22) where each of the left \& the right side is fixed on $\frac{1}{2} m_{o} c^{2}$ which when be added together we can get a description of a rest particle.

4) After all, Equation (22) leaved behind; $n=c_{o} n_{o}$ which means relativistic replication (self-repetition) with the same density to conserve the initial condition $\left(r=r_{o}\right)$.

5) If a particle moved in speed $v$ (the motion inside our sphere comes from Equation (10)) then the linear mass density; $\frac{m}{r}=\left(\gamma m_{o}\right)\left(\frac{\gamma}{r_{o}}\right) \equiv\left(\gamma^{2} m_{o}\right) \frac{1}{r_{o}}$.

So, this may carry a hidden relativistic meaning appearing as creation of new rest particles (If-and only if-we were inside Equation (2)) counting $n=\gamma^{2} n_{o}$. 
Equation (22) goes straight forward to realize the initial condition so it defined well the alternative relativistic solution as in Equation (28).

6) The strategy of Equation (22) is that; since Equation (2) is the final macro state so $m_{o} \& r_{o}$ of Equation (22) should be eliminated from co where each side of the equation is equivalent to $\frac{1}{2} m_{o} c^{2}$.

7) We have to notice that the done algebraic simplification makes $u_{G}$ of Equation (22) \& (23) not thoroughly expressing the relativistic gravitational energy described by Equation (14), so it is rather the alternative relativistic gravitational rest energy describing (with the alternative relativistic electric potential $u_{e}$ ) the rest particle inside the sphere. Also we have to notice that creation of particles here is relativistic (not by collision) self-repetition.

8) Because the term $u_{G}(\gamma)$ is a function in $\gamma$ it is relativistic. And because it is derived from the packet of all the above equations as a function in the gravitational energy $u_{G}(\gamma)=u_{g} \gamma^{2}$ so it is gravitational. But because it is derived from the same equations as; $u_{G}(\gamma)=u_{g} \gamma^{2}=G \frac{m^{2}}{r_{c} n^{1 / 3}}\left(n \gamma^{2}\right)$ so it is not absolutely the typical potential. Thereafter, we should call it 'the relativistic gravitational rest energy which with its conjugate: $u_{e}$ (or $u_{c}$ as we will see) construct the rest energy of the system $u_{G}+u_{e} \equiv m_{o} c^{2}$. Here we must discriminate between the term $u_{G}$ and the alternative relativistic gravitational potential $u_{g}(N)$ which is alternative relativistic because it is a function in $N=n \gamma^{2}$ while it is potential because it is written in the form $u_{g}(N)=-G \frac{m_{2}}{r_{c}\left(n \gamma^{2}\right)^{1 / 3}}\left(n \gamma^{2}\right)$. Here you notice that it is a negative term with denominator formed of the real radius of the relativistic sphere (you will see astronomic application in Equations (74)-(76)). The rest energy, all over the article, means the additive conjugate $u=u_{G}+u_{e} \equiv m_{o} c^{2}$ which is the energy of the system when be distributed all over the particle which means rest energy per a particle which, consequently, when added to the inertial internal rest energy of the particle we get equation 25 which is equivalent to (27) which, consequently realizes (20) whose negative sign is absorbed physically in (28) to realize, finally, the condition $m=m_{o}$ which realizes Equation (1) which means abortion of the kinetic energy of the system.

9) The particle is now defined (after the additive process) by the rest state $(0+$ 0 ), which means no motion and so no relativistic relation. To fire the relativistic relations we defined the Compton rest state of the particle to appear as; $m_{o}\left\{c^{2}-\varphi\left(u_{g}\right)\right\}+m_{o} \varphi\left(u_{g}\right)$ such that the contents of the outer brackets of the left term when be add to the squared speed arising from the gravitational energy we get complete rest state (neither above nor below). The squared speed $v^{2}=c^{2}-\varphi\left(u_{g}\right)$ is the one which fired out each of the relativistic relations $\&$ the micro-events which we studied in all the above equations. But these microstates are constrained by the final rest state: $m_{o}\left\{c^{2}-\varphi\left(u_{g}\right)\right\}+m_{o} \varphi\left(u_{g}\right) \equiv m_{o} c^{2}$.

10) Equation (25) does not mean neither description of two particles nor 
production of a new particle where (25) is defined by (27). So self repetition means neither transformation of energy into new particle nor creation of particles by collision. But it means-here-alternative relativistic solution.

11) If we treated our sphere as in the semi-empirical model then the above is the coulomb's term while the others would be discussed step by step.

12) After all, Equation (2) does not add a new particle, but it describes (new) rest energy originating from the Compton's sphere (per a rest particle) exactly as equation 1 describes the rest mass of the rest particle.

13) Since the electromagnetic energy density describing the rest particles is constant and does not obey the superposition (if we have a proton among a collection of protons inside a sphere, then each proton is still possessing rest energy defined by $E_{o}=m_{o} c^{2}$ whatever the central electric energy of the collection may be), and because each of the conservative energies here is describing rest energy so it is-also-constant \& does not obey the superposition. Hence, once each side of the equation-which is describing the rest particle-, is equal $\frac{1}{2} m_{o} c^{2}$ then it would not obey the superposition. If the left side of the equation went straightforward with the conservative electric energy then it had to be multiplied by $P$ not $p$ but since it became describing the rest energy of the particle so $p$ which realizes $E_{o}=m_{o} c^{2}$ is the correct. If this answer was not enough and if we asked about the fate of the electric energy of the ratio $\frac{P}{p}$ then we can give the next explanation. Put $P=p c_{o}$ instead of $p$ in the nominator of the left side of the equation then $u_{e}=\frac{1}{2} m_{o}\left(c^{2} c_{o}\right)$.

Although this is contrasting with the current knowledge but let us go on to see that our solution refuses this incorrect speed. Now we want to see the relativistic effect of the speed $\left(c^{2} c_{o}\right)$ on the number of the particles $P$ so it is considered rest number, so if we put the relativistic number $P_{r}$ inside the alternative relativistic solution (28) and since the initial conditions-which appear in (27)-then we get;

$$
m^{2} P_{r}=\frac{P m_{o}^{2}}{1-\frac{c^{2} c_{o}}{c^{2}}}=\left(-m_{o}^{2}\right) \frac{P}{c_{o}}=\left(-m_{o}^{2}\right) p \text { Such that } c_{o} \gg 1
$$

where, the minus sign has a final physical meaning realized by Equations (25) \& (27). This means that the alternative relativistic solution returns $P \rightarrow p$ \& $E_{o}=m_{o} c^{2} c_{o} \rightarrow E_{o}=m_{o} c^{2}$. This is a critical point and should be understood well, otherwise it leads to misunderstand. Equation (1) describes $E_{o}$ of the rest (not the relativistic) particle. Each $E_{o}$ defines a certain space and does not traverse to the space of the other particle. Since Equation (1) \& (2) are synonym then, $u_{e}+u_{G}=u \equiv E_{o} \equiv m_{o} c^{2}$ and again these $u(\mathrm{~s})$ do not traverse to each other. And again, since (1) \& (2) are synonym then replication of the particles means also replication of these conjugates $(u)$, which means repetition of the Compton's 
sphere as a copy \& paste (with the same density of matter \& the same particle rest energy). By other words, each $u=E_{o}=0.752 \times 10^{-10} \mathrm{~J}$ (the 'physical secretion' of the particles) is blocked \& inhibited (and so, not traverses each other). The conjugate $u_{e}+u_{G}$ after adding $u_{g}$ (as it is represented in Equation (8)) where Equation (2) is fixed well on Equation (1) we get the same particle (which is defined by Equation (1)) with the new additive $r_{c}$ definition (as in Equations (25) \& (27)). Explicitly, energies of the sphere-itself-appear as summation of quantities, each is equivalent to $m_{o} c^{2}$ (rest form) which when be added to $m_{o} c^{2}$ of the particle realizes the entire equations of Compton's sphere.

This addition-after the physical engulf of the negative sign (which appears with $m^{2}$ in the original relativistic solution) by Equation (22)-leaves behind the relation $m=m_{o}$ hence, we need alternative relativistic solution. This appears as repetition of Equation (1) by the repetitive factor $c_{o}=\gamma^{2}$

14) The physical meaning of each of the new extra definition of the rest macro-sate of the particle (inside our sphere) which is defined by $r_{c}$ and the physical meaning of the imaginary factor which is plugged in Equation (21) would be more clear from the next study of the wave function of the particle. The particle inside our sphere is defined by $c^{2}-\varphi\left(u_{g}\right)$ which describes a real motion (oscillation) and also defined by, $c^{2}-\varphi\left(u_{g}\right)+\varphi\left(u_{g}\right)=c^{2}$ which is the macro-state as explained by Equations (1), (2), (26) \& (27).

Since solution of Schrödinger Equation (1D) for a particle inside infinite potential well takes the form of, $\Psi=A \sin (k x)+B \cos (k x)$ where $B \cos (k x)$ is a zero term

$$
\therefore \Psi=A \sin (k x)=A \sin \left(\frac{p}{\hbar} x\right)=A \sin \left(\frac{m v}{\hbar} x\right)
$$

a) Speaking about the relativistic function and the real speed of the particle then we would have the alternative relativistic relations defined by Equation (28). Therefore,

$x=x_{o}, \quad m=m_{o} \& m n=m_{o}\left(\gamma n_{o}\right)=m_{o}\left(n_{o} \sqrt{c_{o}}\right)$. The factor $\sqrt{c_{o}}$ is integer acts-mathematically-on the angle $\theta$ of the above function as a repetition factor. So the relativistic wave function appears as, $\Psi=A \sin \left(\frac{m v}{\hbar} x\right)=A \sin \left(\frac{\sqrt{c_{o}} m v}{\hbar} x\right)$. $\therefore$ The function returns again to its common form; $\varphi=A \sin \left(\frac{m v}{\hbar} x\right)$ which runs straight forward with the common solution. $\rightarrow\langle x\rangle=\frac{1}{2} l=r_{c}$

This solution treats the particle as a point moves really forward from 0 to $L$ and backward in the opposite direction. But finally the particle looks in the mid-point. It is better to say that $r_{c}$ carries here the meaning of the spacing not the rest radius of the particle (The energy density is explained in a next section).

b) The next goal is to show that the zero term of the wave function solution has physical meaning for the particle inside our sphere. So, we would search in 
how this zero term (of the wave function, $1 D$ ) realizes the new extra definition of the rest particle inside our sphere. This extra definition needs describing the relativistic mass by (26) where the imaginary factor appears as, $m=i m_{o}$ to describe the rest energy state defined by (27).

$\therefore \Psi=A \cos (k x)=A \cos \left(i m_{o} \frac{v}{\hbar} x\right)=A \cos (i k x)$. From the trigonometric identity;

$\therefore \cos (i \theta)=\frac{1}{2}\left(\mathrm{e}^{-\theta}+\mathrm{e}^{\theta}\right)$. To solve this equation we need to understand the physical meaning of this case. If the case satisfies no motion then $x$ is constant and the integration is transformed into simple multiplication.

$$
\therefore\langle x\rangle=A^{2} \int_{0}^{L} \Psi^{*} x \Psi \mathrm{d} x \equiv \frac{A^{2}}{4} x \sum_{\Delta l}\left(\mathrm{e}^{-\theta}+\mathrm{e}^{\theta}\right)^{2}=\frac{A^{2}}{4}\left(\mathrm{e}^{-\theta}+\mathrm{e}^{\theta}\right)^{2} l
$$

After normalization of $\frac{A^{2}}{4} \sum_{\Delta l}\left(\mathrm{e}^{-\theta}+\mathrm{e}^{\theta}\right)^{2}$

$$
\therefore\langle x\rangle=l
$$

The solution tells us that if 1 is the length of the particle then its expected length is 1 .

Although it is a trivial solution yet it realizes Equations (26) \& (27).

This means that the first term $\sin (\theta)$ of the wave function solution, first, describes the micro-event of the particle which is defined by the real motion $\left\{c^{2}-\varphi\left(u_{g}\right)\right\}+\varphi\left(u_{g}\right)=v^{2}+\varphi\left(u_{g}\right)=c^{2}$ where the contents of \{\} brackets represents this motion and gives the relativistic alternative solution defined by Equation (28), and second it realizes the alternative relativistic solution (Equation (28)) where the repetition factor appears mathematically here as repetition of the angle $\theta$. While the zero term of the same wave function realizes its rest macro-state-where $\left\{c^{2}-\varphi\left(u_{g}\right)+\varphi\left(u_{g}\right)\right\}=c^{2}$ represents the rest energy-and hence, the imaginary factor of Equation (26) is engulfed physically and finally appeared as a real physical meaning.

15) The last point here is to know how the original relativistic solution takes place in the radial direction. If the motion of the sphere's particles occurs in all directions in the same time (like explosion or growth) then we can write the invariant event interval $\mathrm{ds}^{2}$ as follow.

$$
\mathrm{d} s^{2}=c^{2} \mathrm{~d} t^{2}-\mathrm{d} r^{2}=c^{2} \mathrm{~d} t^{2}-\mathrm{d} x^{2}-\mathrm{d} y^{2}-\mathrm{d} z^{2}=c^{2}\left(\overline{\mathrm{d} t^{2}}\right)-\left(\overline{\mathrm{d} x^{2}}\right)-\left(\overline{\mathrm{d} y^{2}}\right)-\left(\overline{\mathrm{d} z^{2}}\right)
$$

where the primed terms stand for the moving coordinates. We can formulate the relativistic relations of the motion as follow.

$$
\begin{aligned}
& c \overline{\mathrm{d} t}=\gamma c \mathrm{~d} t-\gamma \mathrm{d} x \frac{v_{x}}{c}+0+0 \\
& \overline{\mathrm{d} x}=-\gamma \mathrm{d} t \frac{v_{x}}{c}+\gamma \mathrm{d} x+0+0 \\
& \overline{\mathrm{d} y}=-\gamma \mathrm{d} t \frac{v_{y}}{c}+\gamma \mathrm{d} y+0+0
\end{aligned}
$$




$$
\overline{\mathrm{d} z}=-\gamma \mathrm{d} t \frac{v_{z}}{C}+\gamma \mathrm{d} z+0+0
$$

And finally, we can recall the properties of the metric tensor and write: $\overline{(r)^{2}}=\gamma^{2} r^{2}$.

Again, because aborting this original solution, then, the alternative one should take place.

After all, we saw a "physical snake" have engulfed the repulsive electric energy which appeared with the relativistic $u_{G}$ as a conjugate pair. Once the initial condition was realized then we need a relativistic solution-other than the original one-which appears as Equation (30). Microscopically, the events inside the Compton's sphere are fired up by Equation (7) (where the real velocity $v=c+$ deficit) or by the big brackets of Equation (6) the alternative relativistic solution passing through the entire packet of the equations from 7 till 68 and finally it should appear in the form of Equation (1). Since, the macro-state of the relativistic Compton's sphere is no more than multiplication of equation one by the number one so you will notice that the macro-state of the entire packet of the equations from 1 till 96 (which describes the interior of the giant proton) obeys-macroscopically-the same rule (conservation of density of the states \& density of matter, and so it describes forever the rest state).

Actually the sphere gives the solution as growth in the three dimensional space, with the final constant density, from the initial sphere with the initial number of particles $n$ and $p$-let us call it the giant charge unit (GCU) - till it reaches to the final sphere (let us call it the giant charge GC) with the final number of particles $N$ and $P$. Or; (GCU) $c_{o}=(\mathrm{GC})$. Hence, the goal-in this point-is to define astrophysical meaning for $N$ such that the giant charge is fit to orbit. We need to understand the transformation of energy (actually, - as we will see-it is not passive transformation but it is relativistic solution) into matter in this alternative solution deeply and not superficially. We need the entire equations packet; we need the particles spacing $r$ to be defined by a bit excess above $r_{c}$ which gives quantum speed defined by a bit quantity below $c$. Also we need to pick up the relativistic imaginary solution and deal mathematically with it to find that $m=m_{o}$ once we found that the relativistic mass equal the rest mass then it is the beginning to choose the alternative instead the original solution. Before all the above, we need to define the speed-of particles-which depends on the unknowns $\mu \& c_{o}$ which should be defined as we will see. Actually, the macroscopic picture of our sphere would be clear step by step. As we saw that $u_{G}$ absorbs-physically-the repulsive effect of $u_{e}$ also in the next chapter we will see that it absorbs the objections of the quantum mechanics. Now the GCU is formed of two distinguishable particles; the protons $p$ and the neutrons $n$ where $n \gg p$. So, each of both has its specific distinguishable potential (inertial) well [3]. We saw that the proton in its potential well appeared as a pair of conjugate. Also the neutron does, so we are still scoping the micro-events. 


\section{The Relativistic Neutrons Potential Well (Asymmetry Term)}

Let us define the Compton's (reduced) wave length of a free neutron as; $r \approx 2.09 \times 10^{-16} \mathrm{~m} \quad[3]$.

And let the momentum to be defined as: $\hbar=m v r$ where $v$ is the speed defined by Equation (10), so let us incorporate this length in our sphere.

To define the spacing which defines this $v$ we have to consider two factors, the first is: since the particle is not free but it is in a spherical collection so we have to define the energy density inside this sphere, and the second is the uncertainty effect. Since we need to realize the maximal possible expected speed which is just a bit beneath $c$ then we expect that the spacing is actually a bit over $r$.

The uncertainty of the reduced Compton's wave length means transient transformation of matter into energy and vice versa or actually creation and annihilation. This is not our case, where self-replication in our Compton's sphere is a relativistic action. This means that if the uncertainty $\Delta r=\frac{1}{2} r$ then the spacing $r_{c}=r+\Delta r=1.5 r$ that is because the particle in our sphere is forbidden to have spacing below $r$ otherwise it would not be matter. So finally we divide this spacing over the factor defined by Equation (10) as:

$$
r_{c}=1.5 r \div \sqrt{1-\frac{n}{p \times \mu}}=1.5 r \approx 3.135 \times 10^{-16} \mathrm{~m} \quad \text { (where, the factor under the }
$$
root-quantitatively_is negligible as we will see).

1) Since the highest energy which anybody can acquire is $\frac{1}{2} m v^{2}=\frac{1}{2} m c^{2}$ then the exclusion quantum energy (symmetry term) of any collection of particles (inside a closed system) never exceeds this highest energy and consequently its spacing never exceeds the above length. In the next discussion we will search in the exact required density which realizes the highest possible energy inside Compton's sphere where the initial conditions are realized.

This density allows us to use the new expression; Compton's sphere instead of Fermi sphere. We expect that the density inside the sphere is in this range but the accurate value of the spacing should be defined from the density of the states of the collection. In GCU and because of $n \gg p$ Pauli's term $u_{c}$ of the Compton's sphere appears as predominant positive kinetic energy. For each neutron we have asymmetric (Pauli) term [4] on the same principle of the liquid drop model of the nucleus in the form of; $u_{c}\left(A-2 z^{2}\right) / A=u_{c}(n-2 p) / n=u_{c}$

(You noticed that number of nucleons $A$ is given here in our model the symbol $n$ and number of the protons $Z$ takes here the symbol $p$ )

In the Compton's sphere (on the same way of Fermi sphere) [5]; Pauli's exclusion Principle needs to multiply the count of the filled states by two (+/-spin). We need-also - to divide over eight [5] to get only the positive space octant (where $\sin -k x=-\sin k x$ ). So, we have the density of states $=n_{s} / V=$ density of the neutrons $=n / V=$ the inverse of the infinitesimal volume [6], and we have 
(on the same way of mathematics of Fermi sphere) the quantum energy density (which appears here in our model as Pauli's term because of $n$ is very much more than $p$ ):

$$
u_{c}=\frac{\hbar^{2}}{2 m}\left(\frac{3 \pi^{2}}{V} n\right)^{2 / 3}
$$

This-for all neutrons-becomes

$$
\int_{0}^{n} u_{c} \mathrm{~d} n=\frac{\hbar^{2}}{2 m}\left(\frac{3 \pi^{2}}{V}\right)^{2 / 3} \int_{0}^{n} n^{2 / 3} \mathrm{~d} n=\left[\left(\frac{3}{5}\right) u_{c} n\right]
$$

where $n$ is the quantity of the neutrons of the giant proton unit and $V$ its volume. And finally divide over $n$ to get the average $\left\langle u_{c}\right\rangle=(3 / 5) u_{c}$

By putting $r_{c} \approx 3.135 \times 10^{-16} \mathrm{~m}$ and by substitution in the above equation, we can estimate the average; $\left\langle u_{c}\right\rangle=0.748 \times 10^{-10} \mathrm{~J} \approx \frac{1}{2} m_{o} c^{2}$.

This is a comfortable result, but since the accurate value of $\frac{1}{2} m_{o} c^{2}=0.752 \times 10^{-10} \mathrm{~J}$ and since our search in this point is not to define $\left\langle u_{c}\right\rangle$ at $r_{c}=3.135 \times 10^{-16} \mathrm{~m}$ but is to define the required spacing at $\frac{1}{2} m_{o} c^{2}=0.752 \times 10^{-10} \mathrm{~J}$ therefore by substitution of $\frac{1}{2} m_{o} c^{2}=0.752 \times 10^{-10} \mathrm{~J}$ in (31) we can get

$$
\left\langle u_{c}\right\rangle=0.752 \times 10^{-10} \mathrm{~J} \equiv \frac{1}{2} m_{o} c^{2} \text { at the spacing } r_{c}=3.127 \times 10^{-16} \mathrm{~m}
$$

2) This is the accurate spacing which defines the Compton's sphere (as defined by Equation (10)). You can notice that the major character of this sphere is that the in-between distances are in the range of the reduced Compton's wave length $r_{c}$ so you can take a Fermi sphere and put inside it the density as $r=r_{c}=3.127 \times 10^{-16} \mathrm{~m}$ and then divide each $r_{c}$ over $\gamma$ (or over $\sqrt{c_{o}}$ ) and multiply each $m$ with the same factor. Let us do, under the initial conditions and by the same rules of the proton potential well.

The relativistic gravitational energy $u_{G}(v)$-as speed function-would be affected by the speed $v$ more than the quantum term field $u_{c}(v)$ such that the factor $c_{o}$ would appear in $u_{G}(v)$ with power $3 / 2$ while it appears in $u_{c}(v)$ only with power $1 / 2$.

If we write $V=n \frac{4 \pi}{3} r_{O}^{3}$ and if we put the factor $A$ instead of the result of multiplication of the constant numerical factors $\hbar^{2} / 2\left(3 \pi^{2} \div \frac{4 \pi}{3}\right)^{2 / 3}$ in Equation (31) then we can rewrite Equation (31) after simplification as follow;

$$
\therefore u_{c}=A \frac{c_{o}}{r_{o}^{2} m_{o} c_{o}^{1 / 2}} n^{2 / 3}
$$

The factor $c_{o}^{1 / 2}$ arises from the relativistic mass in the dominator, while the factor $c_{o}$ arises from the relativistic squared length $\left(r^{3}\right)^{2 / 3}$. The relativistic 
gravitational rest energy-as in the previous sections appears as

$$
u_{G}=-G n^{2 / 3} m_{o}^{2} c_{o} \frac{c_{o}^{1 / 2}}{r_{o}}
$$

where, $A \& G$ are constants and $r_{o}$-here-in these relativistic relations means the rest distance $r_{c}$ (otherwise it means the classic radius of the neutron).

Putting $m_{o}^{2}=-m^{2}$ (please recall in your mind the Equations (14)-(27) in Equation (34), and then equating $u_{c}$ and $u_{G}$ with simplifying $c_{o}$ in both sides we can get

$$
\frac{A}{r_{o}^{2} m_{o}}=\frac{3}{5} \frac{G m^{2}\left(n c_{o}\right)}{n^{1 / 3 r_{o}}}
$$

Putting $n^{1 / 3 r_{o}}=r_{s}=$ radius of the sphere, we find $u_{G}$ of (34) looks as (22)

$$
\therefore \frac{A}{r_{o}^{2} m_{o}}=u_{G}=\frac{3}{5} \frac{G m^{2}\left(n c_{o}\right)}{r_{s}} \equiv \frac{1}{2} m_{o} c^{2}
$$

Adding the two energies of (36) we get

$$
u=u_{c}+u_{G}=\frac{1}{2} m_{o} c^{2}+\frac{1}{2} m_{o} c^{2}=m_{o} c^{2}=m c^{2}
$$

where; $u_{c} \& u_{G}$ appears as conjugate pair which when be added together then added to the inertial rest energy they realize the state $v^{2}=2 c^{2}$ as a relativistic solution for; $m_{o}^{2}=-m^{2}$ where the negative sign gives the same radical physical meaning (again, the microstates should be chosen to realize the rest macro-state). Here you may notice that we have two frame levels. The proper one in which we see the microscopic events as relativistic $m$ and $r$ obeying the proper rules of the special relativity. The second is the alternative one in which the kinetic energy appears as reproductive energy and consequently, the relativistic mass $m$ appears as $m_{o}$ and $r$ appears as $r_{o}$

The alternative solution is constrained by; inter-between distance $\approx 3.127 \times$ $10^{-16} \mathrm{~m}$. This gives (in the original solution) quantum kinetic energy for each neutron $\approx 0.752 \times 10^{-10}$ joule which when is added symmetrically to its conjugate gives the state of (37). The neutron (like the proton) appears in its potential (or precisely, the inertial) well as a pair of conjugate. We also notice-from Equation (1)-that, $m=m_{o} \rightarrow r=r_{o}$.

\section{The Integral Form of Compton Sphere}

The average of the quantum term as estimated before appears as:

By substitution and putting the value of $r_{c}$ we can estimate the average:

$$
\left\langle u_{c}\right\rangle=0.752 \times 10^{-10} \mathrm{~J} \equiv \frac{1}{2} m c^{2}
$$

But the average of the electric potential energy needs to do the following:

$$
u=k e^{2} \int_{0}^{p} \frac{p_{(r)} \mathrm{d} p}{r}
$$




$$
\begin{aligned}
& p_{(r)}=p\left(\frac{r}{R}\right)^{3} \& \mathrm{~d} p=3 p \frac{r^{2}}{R^{3}} \mathrm{~d} r \\
& \rightarrow \int_{0}^{p} \frac{p \mathrm{~d} p}{r}=3 p^{2} \int_{0}^{R} \frac{r^{5}}{r R^{6}} \mathrm{~d} r=\frac{3}{5} p^{2} \frac{R^{5}}{R^{6}}=\frac{3}{5}\left(\frac{p^{2}}{R}\right) \\
& \rightarrow u=\frac{3}{5} k \frac{e^{2} p^{2}}{R}
\end{aligned}
$$

We can do the same manipulation with the average gravitational energy. So the average gravitational and electric energy appears as

$$
\frac{3}{5} G c_{o} \frac{m_{o}^{2} n^{2 / 3}}{r_{c}}=\left\langle u_{G}\right\rangle=\left\langle u_{e}\right\rangle=\frac{3}{5}\left(\frac{1}{2} m_{o} c^{2}\right)
$$

This form is not compatible with the inertial form $\frac{1}{2} m_{o} c^{2}$ so we can modify the form of the equations to appear as

$$
\frac{3}{5}\left(\frac{r_{o}}{r_{c}} \frac{p^{\frac{2}{3}}}{\left(\frac{n}{p}\right)^{\frac{1}{3}}}\right)=\frac{1}{2}
$$

where, the question is to find the quantity of the protons $p$, which realizes this modified integral form. And to appear as

$$
\frac{3}{5} G c_{o} \frac{m_{o}^{2} n^{2 / 3}}{r_{c}}=\left(\frac{1}{2} m_{o} c^{2}\right)
$$

where, the question is to find $n$ which realizes this modified integral form. But we do not have-from the system-enough equations to define all the unknowns of the system. Let us search.

\section{Definition of the Giant Proton Unit \& Driving Its Unknowns}

From the definition of $\mu$ and from Equation (29):

$$
\mu=c_{o} \frac{n}{p}=\frac{c_{o} n}{p}=\frac{N}{p}
$$

where $N$ is the quantity of the neutrons in the giant proton while $n \& p$ is the quantity of neutrons \& protons in the giant charge unit.

We want to define $c_{o}$ as a universal constant. In the second part of the discussion we will estimate $N$

$$
N=3.009 \times 10^{45} \text { neutrons }
$$

By dividing each side of Equation (22) over $c_{o}$ we can define

$$
\frac{1}{2} m c^{2} \frac{n}{p \cdot \mu}=\frac{1}{2} m c^{2} c_{o}=\frac{u_{G}}{c_{o}}=u_{g}=\xi
$$


And from the meaning of self-replication of the giant proton unit-till it reaches to the giant proton-and recalling Equation (30):

$$
\begin{gathered}
N / n=P / p=c_{o} \\
\therefore N=n c_{o}=\left(\frac{n}{p} p\right) c_{o}
\end{gathered}
$$

And since,

$$
\frac{1}{2} m c^{2}=u_{g} c_{o}
$$

And since,

$$
u_{g}=\frac{3}{5} G \frac{m_{o}^{2} n^{2 / 3}}{r_{c}}
$$

From (22) (24) and (48):

$$
\frac{1}{2} m c^{2}=u_{G}=\frac{3}{5} G c_{o} \frac{m_{o}^{2} n^{2 / 3}}{r_{c}}=u_{e}=\frac{3}{5} k e^{2} \frac{p}{r_{c} n^{\frac{1}{3}}}
$$

By dividing $u_{G}$ of (49) over (46) - where $c_{o}$ is cancelled-we can get the value of $n$.

By putting the value of $n$ and substitution in (46) we can define $c_{o}$ and then from (49) we can define $p$ (from $u_{e}$ ).

From defining $p \& n$ we can define the ratio $\frac{n}{p}$.

From the defined values of $\frac{n}{p} \& c_{o}$ we can estimate $\mu$ from: $\mu=c_{o}\left(\frac{n}{p}\right)$.

At last from (39) we can-also-define- $u_{g}$.

This arrangement in derivation of the unknowns (where we derive $u_{g}$ after solving the entire unknowns) is necessary (we will explain this latter). Now we can estimate all the unknowns as

$$
\begin{gathered}
p=2.431 \times 10^{9} \\
\frac{n}{p}=1.2123 \times 10^{12} \\
n=2.947 \times 10^{21} \\
c_{o}=10^{24} \times 1.021 \\
\mu=1.237 \times 10^{36} \\
v^{2}=c^{2}\left(1-\frac{1}{10^{24} \times 1.021}\right)
\end{gathered}
$$

It is clear-now-that we cannot realize Equation (2) with speed less than that defined by Equation (54). A lesser one cannot realize Equation (6). It is the familiar to equate the particle speed by real number: $v=0, \frac{1}{2}, 10$ and so on but here, physics of the alternative relativistic solution allows equating the squared speed as $c$ minus an infinitesimal cut off. This cut should not allow a 
lesser squared speed than that of Equation (54). But a bigger one (approaching more to the speed of light) is allowed. This allows-from Equations (23) and (56)-a bigger $\gamma^{2}\left(c_{o}\right)$ which means replication of a bigger sphere (this is the scientific base for a more complicated giant atom as we will see later). Therefore, we can define $c_{o}$ to include more complicated giant system:

$$
c_{o} \in \mathbf{R}: \infty>c_{o} \geq 10^{24} \times 1.021
$$

We have to notice that the protons have the same wavy behavior and so it has-also- $u_{c}$ but this term could be neglected because of the distance between two neighbor protons $=10^{4} r_{c}$ (where $n / p$ as we will see is about ten power twelve). So, the exclusive Pauli's term of the proton well appears as; $u_{u}=10^{-8} u_{c}=10^{-8} u_{e}$ so the final count of the protons-which realizes Equation (1) in its coulomb form-is $p=2.431 \times 10^{9}-10^{-8} \times 2.431 \times 10^{9}=2.431 \times 10^{9}$.

Because we estimated the above values from outside the system of the giant unit (using, $N$ ) so we have to test them. So putting the above values in the equations of the system we find finally:

$$
u_{G}=u_{e}=u_{c}=0.752 \times 10^{-10} \mathrm{~J}=\frac{1}{2} m c^{2}
$$

\section{The Deficit \& the Excess as a Perfect Solution}

The goal here is to show how the term $b$ is delivered from the same alternative relativistic solution which was fired by the starting point (Equation (7)). If this term was found independent from the alternative solution and since the deficit equal the excess then both with each other had to give zero term. The zero term would spoil the deficit and consequently aborts the relativistic action. The relativistic action needs a deficit (not zero term). Then, it looks as if the excess was delivered side by side with the term $D$ and by the same alternative relativistic solution. That is to say; Equation (7) is actually the launching point. First, we can summarize what we got.

We "picked up" the imaginary relativistic solution, $m=i m_{o}$ where $i=\sqrt{-1}$ and then converted this imaginary solution into refused solution in the form of $m^{2}=-m_{o}^{2}$.

After that, we introduced this refused solution on the relativistic gravitational energy, and then go on till we reached to the accepted substitutive relativistic solution. So physics of the giant charge could be summarized as a shift from the original solution; $\left(\gamma^{2} m_{o}^{2}\right) n$, into the substitutive (self-replication) solution; $\left[c_{o}(n+p)\right] m_{o}^{2}$.

The alternative solution is surely not passive transformation of energy into matter (not as; $m=\frac{E}{c^{2}}$ ) but surely it is relativistic solution. The passive transformation would give one particle for each of the contributors.

The relativistic solution is completely far from this vision. It could be written as 


$$
m^{2} n=m_{o}^{2}\left(\gamma^{2} n_{o}\right)
$$

In the original solution $n=n_{o}$ while in the alternative solution $m=m_{o} \rightarrow n=c_{o} n_{o}$.

The first point to motivate physics of the Compton's sphere is to insure that the total Compton state final energy $u$, per a particle neither above nor below $m_{o} c^{2}$. Its net value should be exactly $m_{o} c^{2}$. If it was above this limit then this means that $v>C$ which is-in relativity-refused. We need to reach to the highest possible speed because Equation (1) is the subject \& key of the Compton's sphere. But if the particles spacing was equal $r_{c}$ and consequently, the acquired quantum energy $u_{c} \equiv \frac{1}{2} m_{o} c^{2}$ then when it is added with the acquired nonrelativistic gravitational energy $u_{g}$ it would give total acquired energy above $\frac{1}{2} m_{o} c^{2}$ or mathematically, $u>0.752 \times 10^{-10} \mathrm{~J}$ which is refused. Hence, we can write;

$$
u_{c}=u_{e}=u_{G}=\frac{1}{2}\left(m_{o} c^{2}-u_{g}\right)=\epsilon
$$

Since, $m=m_{o}$ (if Equation (27) was realized) then we can describe the particle represented by Equation (2) and also represented by Equation (9) as

$$
E=E_{o}=m_{o} c^{2}=m c^{2}=\left(m_{o} c^{2}+u_{g}\right)-u_{g}
$$

where, $u_{g}$-with the small letter subscript-is stand for the nonrelativistic gravitational energy (not $u_{G}$ ). Equation (58) surely describes (1 \& 2) but-till up now it cannot describe our sphere without realization the condition mentioned in ( 9 \& 27) which are not yet realized. Equation (57) is just to say that: to prevent excess speed over $c$ or to prevent the state which is inside the brackets of (58) we have to add $-u_{g}$ or definitely the acquired energy must be not over the energy of (57). But this equation alone doesn't tell us how does $u_{g}$ precipitate in the system. All the equations which contain a cutoff energy-from Equation (6) and upward - could be understood on this base. In Equation (7) - as example-the deficit $\equiv u_{g}$ so when the system adds $u_{g}$ as in (6) we guarantee that no excess speed over light. Definitely it says; if $m_{o} c^{2}$ represents acquired energy then it is forbidden to add to it any infinitesimal energy so the contents of the above brackets in (58) is forbidden and hence the energy should appear as in (57).

You can have a population of neutral particles with zero electric energy. And you can have a population of particles with zero short range energy if the in-between distance was far from Fermi range. And you may get a population of matter with zero quantum energy such that Pauli's Exclusion Principle may disappear in condensate state (like; Cooper's pair \& neutron pair). Moreover the quantum term which appears in Equation (33) depends reversibly on the mass so it disappears at the mass goes to infinite. Definitely, you can forever multiply a wave function-describing a quantum particle-by a non-zero complex constant A, to get-by linear combination-the same state with the same physical concept as: 
$\psi(x)=A \varphi(x)$. That is to say, linear space itself is a subset of Hilbert space which means that the exclusive efficiency vanishes at linear combination.

But you never get matter without gravitational energy. On general: no field is forever synonym to matter except the gravitational field. Since each of the contributors; $u_{e}, u_{c}$ and $u_{G}$ are energies would be added to the particle and since the gravitational field never vanish from matter and since the system supplies each particle with $u_{g}$ then the contributors should have a deficit equal $u_{g}$ otherwise the added $u_{g}$ of the system would add speed to light which is forbidden. This additive $u_{g}$ is the residual energy of the system after withdrawing all the other energies.

Since, $\left(m_{o} c^{2}-\xi\right)+u_{g}=m_{o} c^{2}$

$\therefore \xi=u_{g}$ If-and only if-the system equations precipitate $u_{g}$

We began with Equation (7) and followed up the microstates of the coulomb's form where each conjugate pair realized in the approximate solution that the relativistic mass is equal to the rest mass, so up till now we are still maintaining the

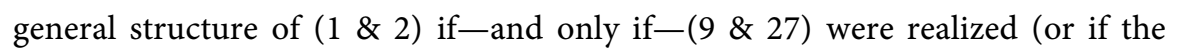
term $b$ was deposited). Equation (58) expected presence of $u_{g}$ but it did not precipitate it. So we need to fix (57) on Equation (58) (but without its brackets). Let us see how. Now, since $v \approx c$

And since, $v^{2}=c^{2}-\frac{c^{2}}{c_{o}}=c^{2}-c^{2} \frac{n}{p x \mu}$.

Then the conjugate pair $u$ appears as:

$$
u=u_{e}+u_{G} \equiv\left(m_{o} c^{2}-u_{g}\right)
$$

Again, since $v \approx c$ then, one can say that the relativistic solution $v^{2}=2 c^{2}$ should also take the form

$$
v^{2} \approx 2 c^{2} \rightarrow v^{2}=2 c^{2}-2\left(c^{2} \frac{n}{p \mu}\right)=2 c^{2}-2\left(c^{2} \frac{1}{c_{o}}\right)
$$

Actually this speed (as we will discuss later on) doesn't need external source of energy outside the system. If we looked to the neutron in its well and if $r_{c}=r$ then it would appear as energy not as matter. But because the inter-space here is just above this limit so-from de Broglie wave-we get oscillatory motion defined by (10). Also the proton does in its well. This is a description of the microstate events. If we took the Equations (20), (24) \& (37) we find them describe the macro state of a system perfectly fixed on (1). That is to say: if Equation (1) describes the rest mass $m=m_{o}$ of the rest particle then its synonym (Equation (2)) describes the rest energy $E=E_{o}$ of the Compton's sphere per each rest particle. This means that if (1) succeeded to arrest its left side to describe-in its right side-the rest mass then also does Equation (2) where it succeeded to arrest its left side to describe-in its right side-the rest energy (of the Compton's sphere per a rest particle). Hence, a deficit (or excess) in (2) should mean necessarily the same deficit (or excess) in (1) and vice versa.

This idea should be maintained and never waste. Explicitly, the energy which 
we observe in a conjugate pair of $u$ is not more than description of the rest energy of the Compton's sphere per rest particle perfectly as Equation (1) describes the rest mass.

Equation (58) describes the rest energy of the particle of Equation (1) perfectly. And it can also describe the particle of our sphere but only when we do approximation for the relation $v^{2} \approx 2 c^{2}$ to appear as $v^{2}=2 c^{2}$.

The approximate solution enabled us to fix our system on the form of (1), where (58) described the rest energy as a perfect synonym to the rest mass.

Now we can begin again from the speed which is defined in (10), so let us recall the accurate value of $v^{2}$ as it is defined in (60) and put it in its original relativistic relation

$$
\begin{aligned}
& \therefore m^{2}=\frac{m_{o}^{2}}{1-\left(2-2 \frac{1}{c_{o}}\right)} \\
& \therefore-m^{2}\left(1-\frac{2}{c_{o}}\right)=m_{o}^{2}
\end{aligned}
$$

As in the approximate solution, we can put here $m_{o}^{2}$ in place of $-m^{2}$ (again, the micro-states should be directed to realize the macro-state, meaning that we are searching, here, for the condition required for the microstate; $v \approx c$ to realize the macro-state; $v^{2}=2 c^{2}$ )

$$
\therefore m_{o}^{2}\left(1-\frac{2}{c_{o}}\right)=m_{o}^{2}
$$

This is the same radical physical meaning of the proposal of (20) where here it again engulfs the negative sign of the equation in which it was introduced to leave behind a physical meaning. The equation-now-tells us that we have one particle with two different rest masses. The unique solution should be defined by different systems. That is to say the right side of the equation represents the rest mass of the particle $\left(m_{s}\right)$ inside the sphere before adding $u_{g}$ while the left side represents $\left(m_{o}\right)$ outside the sphere or inside the sphere after adding $u_{g}$

$$
\therefore m_{o}^{2}\left(1-\frac{2}{c_{o}}\right)=m_{s}^{2}
$$

$\therefore m_{o} \sqrt{1-\frac{2}{c_{o}}}=m_{s}$. Using the binomial theorem

$$
\begin{aligned}
& \therefore m_{o}\left(1-\frac{1}{c_{o}}\right)=m_{s} \\
& \therefore m_{o}-\frac{m_{o}}{c_{o}}=m_{s}
\end{aligned}
$$

By simple simplification and multiplying each side by $c^{2}$ and then substitution from (44) with the value of $u_{g}$ and substitution in (63)

$$
\therefore m_{o}-\frac{m_{o}}{c_{o}}=m_{s} \rightarrow m_{o} c^{2}-\frac{m_{o} c^{2}}{c_{o}}=m_{s} c^{2} \rightarrow m_{s} c^{2}+u_{g}=m_{o} c^{2}
$$


This is the relativistic solution for existence of the nonrelativistic $u_{g}$ in our system. Hence, we can fix (57) on (58) as:

$$
E=E_{o}=m_{o} c^{2}=m c^{2}=\left(m_{o} c^{2}-u_{g}\right)+u_{g}
$$

where the contents between the brackets represent the equivalent energy of the rest mass of the particle inside the sphere (the particle with the deficit) while the positive $u_{g}$ is the added gravitational energy so the relativistic mass $m c^{2}=m_{o} c^{2}$

Also the energy $u_{c}=u_{e}=u_{G} \equiv \frac{1}{2}\left(m_{o} c^{2}-u_{g}\right)=\in$ is defined-now- by:

$$
\begin{gathered}
\left(m_{o} c^{2}-u_{g}\right)+u_{g}=m_{o} c^{2} \\
2 \in+u_{g} \equiv m_{o} c^{2}
\end{gathered}
$$

If we put: $m_{s}=2 \ni \&$ the deficit in each of the mass \& energy respectively as $m_{d} \& m_{f}$

$$
\therefore \in=\ni \& m_{d}=\frac{m_{o}}{c_{o}} \equiv \frac{m_{f}}{c^{2}} \equiv \frac{u_{g}}{c^{2}} \& m_{f}=u_{g} \& m=m_{o}=m_{s}+m_{d}
$$

$\therefore m_{o}=\varphi\left(u_{g}\right)$, therefore it is the relativistic mass of $m_{s^{*}}$

We notice also in the approximate solution that, we used $m=m_{o}$ which gave approximate solution in the form of Equation (27). Here-in the perfect solution-we can discriminate between $m_{o} \& m_{s}$ where the perfect solution appears as in (60). We can clarify the entire physical meaning of all the equations from (22) till (66) as the next:

Since the deficit in the rest mass of the particle which is defined by Equation (1) is a deficit in the rest energy of the Compton sphere (per a particle) which is defined by Equation (2), so we began from:

$$
\frac{1}{2}\left(k \frac{e^{2}}{r_{o}}-u_{g}\right)=\frac{1}{2} m_{s} c^{2}
$$

This deficit fired up the relativistic solution by the speed which is defined by (10). Then we used the original relativistic technical tools which finally produced the alternative relativistic solution which appeared as: $n=\gamma^{2} n_{o}$ where $\gamma$ is defined by the speed which is defined by (10). Beside this relativistic solution we discovered another relativistic solution represented in that: this speed which is defined by (10) does not affect the mass, so the solution was: $m=m_{o}$ but this solution $\left(m=m_{o}\right)$ is defined by a condition defined by (27) which needs adding an excess quantity equals the deficit quantity. We called that solution "the approximate solution" which did not show how such quantity was added. The perfect solution is the complementary solution which explained this point. Since that, then it has to use the same relativistic technical tools but with the correct speed defined by Equation (10). Then we have to allow the proposal defined by (20) to interact mathematically with this correct speed to see the relativistic condition required for producing this excess. The perfect solution discovered that; the alternative relativistic solution is the one which added excess mass and con- 
sequently excess energy equal the deficit. So, we can summarize as

$$
\left(\frac{1}{2} G \frac{m_{s}^{2}}{r_{s}}\right) n c_{o}+\frac{1}{2} G \frac{m_{d}^{2}}{r_{s}}=\left(\frac{1}{2} k \frac{e^{2}}{r_{o}}-\frac{1}{2} u_{g}\right)+\frac{1}{2} u_{g}=\left(\frac{1}{2} m_{s} c^{2}\right)+\frac{1}{2} m_{d} c^{2}
$$

where, the content between the brackets appears with its deficit which fired the alternative relativistic solution which delivered all the outside the brackets. Finally we get:

$$
\frac{1}{2} G m_{o}^{2}\left(n c_{o}\right) / r_{s}=\frac{1}{2}\left(k \frac{e^{2}}{r_{o}}-u_{g}+u_{g}\right) \equiv \frac{1}{2} m_{0} c^{2}
$$

And by the symmetric addition we get: $\frac{1}{2} m_{0} c^{2}+\frac{1}{2} m_{0} c^{2}=m_{0} c^{2}=m c^{2}$.

The last equality was put to show that we are finally in a complete rest state $\left(m=m_{o}\right)$. When the mass $m_{d}$ is added to $m_{s} \rightarrow m_{o}$ therefore we can consider $m_{o}$ is the relativistic mass of $m_{s}$ where this relativistic effect is due to the speed coming from $u_{g}$ not from the speed defined by (10).

Defining the unknowns of the Compton sphere depended on the next idea; since we began from $\frac{1}{2}\left(k \frac{e^{2}}{r_{o}}-u_{g}\right)=\frac{1}{2} m_{s} c^{2}$ and since, $m_{s} \approx m_{o}$ while $u_{g} \ll k \frac{e^{2}}{r_{o}}$.

So, the approximate solution ignored the small deference between these quantities where the equation got; $\frac{1}{2} k \frac{e^{2}}{r_{o}}=\frac{1}{2} m_{o} c^{2}$.

This inconsiderable error which was committed by the approximate solution could not be avoided because each of $m_{s} \& u_{g}$ is unknown (but could be derived after defining the other unknowns)

$$
\therefore m_{s}=2 \ni=m_{0}-m_{d}=m_{o}-\frac{m_{o}}{c_{o}}=m_{o}\left(1-\frac{1}{c_{o}}\right)=m_{o}\left(1-10^{-24}\right) \approx m_{o}
$$

This means that from the quantitative side of view we can put $m_{s}=m_{o}$ and we can do our estimations on this base. Nevertheless, from the qualitative side of view the deference between both is the scientific base of physics of the giant atom so we cannot neglect the physical concept of this deference. Although the perfect solution is the one which discovered the term carrying the excess quantity which we were searching for in the approximate solution yet the approximate solution is-also-necessary and we cannot exclude it because it supplies us with the easy or perhaps the unique mathematic method to derive the unknowns of the giant charge unit. If you put $m_{s}$ of the perfect solution in place of the approximate solution you cannot simply derive the unknowns, while-as you saw-it was easy to do in the approximate solution (Equations (45)-(54)). Minimizing the incorrectness (arising from the difference between $m_{o} \&$ $m_{s}$ )-during estimating the Compton sphere unknowns-depends on important idea. Since we expect that $m_{s} \& u_{g} \ll 1$ while each of the countable unknowns $\gg 1$ so we avoided to derive the unknowns from this little quantity, so we de- 
rived at first $N>10^{45}$ from $u_{g}$ of the giant atom (which is comparatively big quantity) not from $u_{g}$ of Compton sphere and then we derived $n>10^{21}$ from $u_{G}$ not from $u_{g}$ and so on all the unknowns were derived independent from $u_{g}$ By other words, since $u_{g}$ is the smallest so the error factor arising from the difference between $m_{o} \& m_{s}$ would be clear in it, so to avoid this effect on the other unknowns we do not derive them from $u_{g}$.

Anyway, Equation (79) from which we will derive the quantity $N$ shows that the speed resulting from the gravitational energy is so little that we can neglect $m_{d}$

The meaning of $m_{o} \& m_{s}$ appears well from the normalized wave function, where the wave number $k=\frac{2}{l}=\frac{m_{s}}{\hbar} c$.

Appearance of the rest mass as $m_{s}$ in the subsection (13-a) is well perfect because this means $c^{2}$ of the fields split as; $\left\{c^{2}-\varphi\left(u_{g}\right)\right\}+\varphi\left(u_{g}\right)=v^{2}+\varphi\left(u_{g}\right)=c^{2}$ where the contents of the outer brackets gave the relativistic alternative solution defined by Equation (28) while the positive $\varphi\left(u_{g}\right)$ would transform-by its relativistic action $-m_{s}$ into $m_{o}$. But 13-b, does not give the meaning of split of $c^{2}$ because it talks about the rest state. Hence, the physics of (13-b) needs the particle to appear as $m_{0}$.

We can summarize all the above chapters to give the global picture as follows;

Given a particle let be called $\mathrm{P}$, defined by $k \frac{e^{2}}{r_{o}}=m_{o} c^{2}$ (see Appendix) where this equation says; the rest mass is equivalent to the rest energy. The particle in the giant charge committee is defined by rest mass $m_{s} \&$ mass deficit $m_{d}$ but since the rest mass of this equation is synonym to the rest energy of equation 2 which describes the macroscopic state of our sphere so this mass deficit appears also as rest energy deficit. Hence the particle appears as

$$
k \frac{e^{2}}{r_{o}}-m_{d} c^{2}=m_{o} c^{2}-m_{d} c^{2} \equiv 2 \in .
$$

This deficit in energy fired out the alternative relativistic solution defined by (56). But the particle with this deficit is not the particle $P$. Fortunately, the relativistic solution itself is the one which delivered energy excess equal the energy deficit. And again, since the rest energy of the sphere per a particle (defined by Equation (2)) and the rest mass (defined by Equation (1)) are synonyms so we have the same excess in the mass too. Hence the particle appears as $\left(k \frac{e^{2}}{r_{o}}-m_{d} c^{2}\right)+m_{d} c^{2}=\left(m_{o} c^{2}-m_{d} c^{2}\right)+m_{d} c^{2}$. This is the particle $\mathrm{P}$, and the net result is $\gamma^{2}$ times the particles count in the sphere. The perfect solution is the one which produces the alternative relativistic solution while the approximate solution is the one which enables us to determine the unknowns of the Compton sphere. Presence of $u_{G}$ in the system beside $u_{g}$ is not contradictive because the first when added with its conjugate has final relativistic meaning not 
more than (as you see that from the equations) $m=m_{o}$ while the second carries the effect of the gravitational energy. So, we can say that, the first (the relativistic one) carries with its conjugate the meaning of $m_{o} c^{2}$ while the second carries the meaning: $\frac{1}{2} m v^{2}$.

As in the semi-empirical mass model the nucleons inside the nucleus has mass deficit which appears as nuclear binding energy also here. The particle inside the Compton sphere has to pay from its pocket to subscribe as a member in this committee. The sphere looks like a giant particle where its vacuum is absolutely energy empty so the particles to live with each other they have to pay from their masses. We have also do stress on that, the system itself by its nonrelativistic equations cannot precipitate $u_{g}$ because each of Einstein \& coulomb form talks nothing about it although coulomb's form (the microstates) expected its existence. The matter also calls it, but the allowed nonrelativistic equations cannot realize it. So the relativistic equations discovered the trivial solution;

$$
u_{g}-u_{g}=0
$$

But its hidden nontrivial meaning is that; each of the minus \& the positive $u_{g}$ $\&$ its synonym $m_{d}$ is a relativistic solution. The deficit in the rest mass of Equation (1) is equivalent to the deficit in the rest energy of Equation (2) but this deficit by the relativistic action have created final relativistic solution formed of one packet appeared as; reproduction of particles counting $(n+p) c_{o}$ each with final rest mass $=m_{o}$ where the entire particles are living in Compton sphere each with mass deficit and with residual gravitational energy. So, $u_{g}$ of the system, although it is non-relativistic entity yet it is one of the products of the alternative relativistic solution.

The kinetic energy appears-by the alternative relativistic solution-in a rest form. This means that the GCU have appeared after all these relativistic micro-states to realize Equations (1) where it means actually that it came from multiplication of Equation (1) by the number one to give the same solution. The energy looks as if it was turned from the region of the kinetic energy and flashed in the region of the reproductive rest energy with the simple conservative momentum \& energy solution;

$$
\boldsymbol{P}=\boldsymbol{P}_{1}+\boldsymbol{P}_{2}=0
$$

$E=2 \int p \mathrm{~d} v=m 2 c^{2}$ where $v=c=$ constant.

In biology, self-replication takes other form. The chromosome of the cell of the living thing-by metabolism-utilizes the nutrients to construct another chromosome. No creation of particles; neither from energy nor from relativistic action. Number of the input of the particles (of the mother chromosome + of the utilized nutrients) is the same of the output (of the mother chromosome + of the born one). The cell during replication does not gain particles. So the substitutive solution is not clearly suitable here. The dead or even the adult, or accurately the immature or even the old cell cannot replicate even if it was put in a bath of the 
required nutrients or-accurately-even if it was put in the same biologic medium required for replication. Here, there is no mass deficit and consequently no energy deficit. The chromosome acts as a template and appears like $\ni=m_{o} c^{2}$ but without deficit, while the particle of the nutrient appears like $\in=m_{o} c^{2}$ (but without deficit). When they added $\rightarrow m_{o} 2 c^{2}=2 m_{o} c^{2}$.

This view does stress on that $\in=\ni$ in inanimate like animate. But the hidden meaning-here-is that; the symmetric addition occurs at $r$ is fixed on the reduced Compton's wave length without excess. This meaning may be useful in understanding the physical background of some diseases like cancer \& some mutational genetic diseases. This needs further independent study.

\section{The Wave Function \& the Concept of the Particle Speed}

The defined density of matter-inside our sphere-alone can offer quantum speed for the neutron, but it cannot alone define the proton speed which needs defined number of protons with the same defined density. We saw that speed of both particles is defined by Equation (10), but what is the concept of this speed? The energy eigenfunction of the particle inside the potential wells could be written as;

$$
\varphi=A \sin k x=A \sin n \pi x / L=A \sin x(\sqrt{2 m E}) / \hbar
$$

where on normalization: $A=\sqrt{\frac{2}{L}}$.

Since, the momentum operator $\check{p}=-i \hbar \partial / \partial x$

$$
\text { And since, } \begin{aligned}
& \partial \varphi / \partial x=(\sqrt{2} / L) n \pi / L \cos n \pi x / L \\
& \therefore\langle p\rangle=\int_{0}^{L} \varphi^{*} \grave{p} \varphi \mathrm{d} x=(i \hbar / L) \sin ^{2} n L \pi / L=0
\end{aligned}
$$

At first glance this conclusion seems strange. After all:

$$
E=p^{2} / 2 m \rightarrow p=\sqrt{2 m E} n= \pm n \pi \hbar / L
$$

The \pm sign provides the explanation: The particle is moving back and forth, and so there should be two momentum eigenfunctions for every energy eigenfunction, corresponding to the two possible directions of motion. The procedure for finding the eigenvalues of a quantum-mechanical operator $\check{p}$ is to put the correct momentum eigenfunction as:

$$
\sin \theta=\frac{\mathrm{e}^{\mathrm{i} \theta}}{2 i}-\frac{\mathrm{e}^{-\mathrm{i} \theta}}{2 i}
$$

Hence each energy eigenfunction can be expressed as a linear combination of the two wave functions so that $\varphi=A \sin n \pi x / L=\varphi^{+}+\varphi^{-}$

$$
\begin{gathered}
\varphi^{+}=A \sin n \pi x / L=A \frac{\mathrm{e}^{i \theta}}{2 i} \rightarrow p^{+}=n \pi \hbar / L \\
\varphi^{-}=A \sin n \pi x / L=A \frac{\mathrm{e}^{-i \theta}}{2 i} \rightarrow p^{-}=-n \pi \hbar / L
\end{gathered}
$$

From above we conclude that the particle inside the Compton's sphere oscillates back and forth with its speed $v \approx c$. 
From the side of view of special relativity, (if the capital letters stand for the measurements on the moving coordinate) we hold on the synchronous measurement $(T=0)$ for a body moving to the right:

$$
x_{o}=(X+v T) / \gamma=X / \gamma
$$

And for a body moving to the left we would hold:

$x_{o}=(X-v T) / \gamma=X / \gamma$ this means that Lorentz length contraction holds with the same form either the body moves back or forth such that $\gamma$ factor depends on the squared speed of $\gamma$ so $v^{2}=(-v)^{2}$ You can notice that our case differs from Doppler effect, such that we want-here-to measure $x$ relative to $x_{o}$ and $-x$ relative to $-x_{o}$

Since-as we have discussed before-both $x_{o} \& m_{o}$ go straight forward in a linear relation so the same result holds with the relativistic mass.

Actually many experimental trials succeeded to trap ultra-cold neutrons in slow speed. High technology succeeded to trap neutrons with speed of 5 meter per second [7] [8]. If we could to get the least possible speed like that of the giant charge then we may get it. But I can suggest that if we had a universe defined by the Equations (45)-(54) or if we had count of neutrons defined by (52) each with speed $v=\sqrt{\frac{2 u_{g}}{m}}$ or may be defined by Equation (10) then the particles may spontaneously condensate to Compton state.

\section{The Solar System as Giant Atoms}

Now in this second part of the discussion we would suggest that the solar system in one of its primordial historical evolutionary stage had presented as giant atoms. This suggestion would be tested by doing comparison between our estimations about the parameters of the orbits of the giant atom and that of the solar system. The tested parameters of the solar system would be:

Speed and radius of the orbit of each planet, the elliptical orbits of the planets and also we would define the ratio between the mass of the sun to the total mass of the entire planets. So, our work suggested that the solar system had begun its motion as units of giant atoms. Later on, the atomic model disappeared after it left behind the above mentioned parameters to refer to the past existence of the giant atomic model.

Now the giant atom was formed of giant charges, and the giant charge had existence in the form of Compton state. Existence of such state-as we saw from physics of the giant atom-has overcome the following problems; coulomb's force which would not allow existence of much charges in a comparative narrow space like the giant charge, Pauli's exclusion principle; which never allow two typical fermions to exist in the same energy level and the quantum energy density which acts against condensation of matter in such a state. We would see also (after estimating the radius of the giant atom orbit) that the giant charge can orbit as a collection of points in a circle without considerable radiation (not vi- 
olating Larmor's equation).

\subsection{The Hydrogen Atom Like System [9] [10]}

Consider an electron $e$, with a non-relativistic mass $m_{e}$ and a proton $p$, with mass $m_{p}$ orbit each other and consider $\mu$ stand-here-for the reduced mass.

The two body problem could be reduced as one body problem as

$$
\mu=m_{e} m_{p} \div\left(m_{e}+m_{p}\right) \& m=m_{e}+m_{p}
$$

If, $m_{p} \gg m_{e}$

$$
\therefore \boldsymbol{F}=m_{e} \boldsymbol{a}=\mu \boldsymbol{a} \text { where, } \boldsymbol{a}=\boldsymbol{a}_{e}-\boldsymbol{a}_{p}
$$

If, $\boldsymbol{r}=\boldsymbol{r}_{e}-\boldsymbol{r}_{p}$

$$
\therefore k e^{2} \div r=m_{e}\left(v_{e}\right)^{2} \div r_{e}=m_{p}\left(v_{p}\right)^{2} \div r_{p}
$$

The Lagrangian $L_{g}$ could be written as;

$$
L_{g}=\left[m_{e}\left(v_{e}\right)^{2} \div 2\right]+\left[m_{p}\left(v_{p}\right)^{2} \div 2\right]-U\left(r_{e}-r_{p}\right)
$$

The modulus of the angular momentum vector $=L$

$$
\begin{gathered}
L=m_{e} v_{e} r_{e}+m_{p} v_{p} r_{p} \approx m_{e} v_{e} r_{e}=\hbar \\
\boldsymbol{r}_{e}=\boldsymbol{r} m_{p} \div m \approx \boldsymbol{r} \\
\boldsymbol{r}_{p}=\boldsymbol{r} m_{e} \div m
\end{gathered}
$$

The ground state $|0\rangle$ could be described by the Hamiltonian $H$ in time dependent form as;

$$
H=-\left(\hbar^{2} \div 2 m\right) \partial^{2} / \partial x^{2}+(E-U)=i \hbar \partial / \partial t
$$

The solution gives;

The expected radius $\langle r\rangle=5.29 \times 10^{-11} \mathrm{~m}$

The expected velocity $\langle v\rangle=2.18 \times 10^{6} \mathrm{~m} / \mathrm{s}$

\subsection{The Major Atom: A Proposed Inversed Atom}

Although it is a proposed system yet it is the mathematic base of the giant atom system. In the giant electron we can hold the same equations of the giant proton.

Since, the proton count $p$ in the giant proton $=$ the electron count $e$ in the giant electron and since the electron mass $m_{e}=\frac{1}{1844} m_{p}$ where $m_{p}$ is the mass of the proton and if we substituted in Equation (16)

$$
\therefore \frac{n}{e}=1844 \rightarrow \frac{M_{e}}{M_{p}}=1844
$$

where, $\frac{n}{e}=$ the ratio between the neutrons \& the electrons in the giant electron, while $\frac{M_{e}}{M_{p}}=$ the ratio between the mass of the giant electron \& the giant 
proton. Hence we can say that the giant atom is a perfect inversed atom. This chapter is to study the mathematics of this inversed atomic system. So, in the hydrogen atom like-system (h.a.l.s); suppose that we succeeded to bind its electron through imaginary energy with a number of neutrons $=\frac{m_{p}}{m_{e}}=1844$ neutrons. And suppose that this proposed binding energy $\geq$ the total energy between the proton and its orbiting electron such that the neutrons bound electron can orbit as one body. Once we can create this imaginary major atom we will notice that here would be shift of the center of the masses towards the neutron bound electron. There would be (also) respective relative shift from quantum to classic physics. Let us study this inversed atom (i.a.) side by side with (h.a.l.s.) and let us use for the first the capital letters and for the second the small letters.

We did nothing except binding the electron with 1844 neutrons so;

$$
k e^{2} \div r=k e^{2} \div R \rightarrow R=r
$$

From (69)

$$
\begin{aligned}
\therefore \boldsymbol{R}_{p} & =M_{e} \boldsymbol{R} \div \mu \simeq M_{e} \boldsymbol{R} \div M_{e}=\boldsymbol{R} \\
& \boldsymbol{R}_{e}=M_{p} \boldsymbol{R} \div M_{e}=\boldsymbol{R} \div 1844
\end{aligned}
$$

where, $M_{e}$ is mass of the electron which is bound with 1844 neutrons $=1840\left(M_{n} \div M_{p}\right) M_{p}=1844$ as much as the mass of the proton.

And where, $\mu=$ the inverse of $\frac{1}{M_{p}}+\frac{1}{M_{e}}=M_{e} \div 1845=M_{p} \div 1.0005 \approx M_{p}$ $\rightarrow R=R_{p}=r \quad \& \quad v=v_{p}$.

From above we can get

$$
k e^{2} \div r=k e^{2} \div R=M_{p} v^{2}
$$

The angular momentum $L$ obeys:

$$
L=L_{p}+L_{e}=l \hbar .
$$

where, $l$ is an acquired inversed atom level number.

On substitution we get

$$
\begin{gathered}
v=2.18 \times 10^{6} \sqrt{\left(m_{e} \div m_{p}\right)}= \\
l=43
\end{gathered}
$$

This speed is the speed of the orbiting proton in this theoretical inversed atom model. Since the giant atom is inversed atom model so it conserves the same speed.

We can get also

$$
\lambda_{o}=\lambda \div 43 \rightarrow 2 \pi R=l \lambda_{o}=l \lambda \div 43=\lambda
$$

where $\lambda$ is the wave length of Bohr's radius.

\subsection{The Huge Charge}

Here we have to focus on the next equations of the giant atom which supplies us 
with the physical unit of the orbiting giant charge; let us call it the huge charge. The huge charge which appears in the giant atom equations is the smallest unit of the orbiting charge. It has a mass $m_{u}$ and a matter wave length $\lambda_{u}$

$$
\begin{aligned}
& m_{u}=m(n \div p) \\
& \lambda_{u}=\lambda \div(n \div p)
\end{aligned}
$$

where $\lambda$ is the wave length of Bohr's radius.

Its angular momentum would be in the form;

$$
(m n \div p)[(\lambda l \div l)(p \div n)] v=l \hbar
$$

The circumference $S$ of the giant orbit is the summation of the huge waves;

$$
S=2 \pi R=\left(\lambda \frac{p}{n}\right) p
$$

The orbital could be considered as one dimensional infinite square well of length $2 \pi r$ or a model for a one-dimensional box. Since that, we have two particles with different states; $+1 / 2$ spin $\&-1 / 2$ spin occupying the same energy level. So that,

$$
S=2 \pi R=\lambda\left(\frac{p}{n}\right) \frac{p}{2}=\frac{\lambda}{2}\left(\frac{p}{n}\right) p
$$

You can-also-consider that the factor 2 in the denominator is due to that; the wave length is formulated as "a particle in a box model" such that: $2 L=\lambda$ $=$ bohr's wave length.

Since, "in this chapter" $n \& p$ is the number of neutrons \& protons inside the giant proton (not inside the Compton sphere) $\&$ since, $\left(\frac{p}{n}\right)$ is constant and is defined here in the giant proton as it was defined in the Compton sphere by Equation (51), and since $p=\left(\frac{p}{n}\right) n$

$$
\therefore S=2 \pi R=\lambda\left(\frac{p}{n}\right)\left(\frac{p}{n}\right) \frac{n}{2}=\lambda\left(\frac{p}{n}\right)^{2} \frac{n}{2}
$$

where, the factor inside the brackets is constant as defined by Equation (51) (it is the same; in the Compton sphere \& in the giant proton) while $n$ is the number of the neutrons inside the giant proton (which we called in Equation (43); $N$ ).

\subsection{The Mathematic Analysis of the Giant Atom Orbit}

The giant proton to orbit the giant electron we need equation in the form of:

$$
G\left(\frac{m \cdot n}{r}\right)\left(\frac{n}{p} \cdot m\right) \geq k e^{2} \frac{p}{2 R}
$$

where the left side of this relation means the gravitational energy inside the giant proton per a huge proton lying on the most outer layer of the sphere (defined by the mass $\frac{n}{p} \cdot m$ ) while the right side is the electric energy between the giant electron and the giant proton in its orbit defined by the orbit radius $\mathrm{R}$ per a huge 
charge. This form simply and directly means that:

Since the spheres are in orbits then the total energy equals half the electric potential energy. If this total attractive electric energy between the sphere A and a huge charge of the outermost layer of the sphere $B$ was just equal or lesser than the gravitational energy which binds this huge charge with its sphere then the sphere would orbit as one body. Obviously, we take a charge from the most outer layer because according to Gaussian's theorem, although the most outer layer has the maximum flux yet its particles has the minimum gravitational potential so we guarantee that if this particle preserved on its sphere then all the particles of the sphere would do the same. If the left side of the above relation was lesser than the right side then the charges of the giant atom would be attracted as singled particles which would lead to evacuating it from the charges and consequently destroying of the whole electric building. This is very important point which would give radical and dramatic event would be discussed later (during talking about the end of Compton sphere). The hidden important meaning behind the factor $\left(\frac{n}{p} \cdot m\right)$ is that we want to guarantee-under the above mentioned condition - that the huge proton is the unit of the orbit.

In one word this condition (the equality form) guarantees that the sphere would orbit as huge charges units with the least amount of $\mathrm{n}$ and consequently of $p$. But the relation in its inequality form can allow additional $\mathrm{n}$ as we will see when we speak about the astronomic giant proton. (Obviously, we can conclude the same required condition if we applied correctly the physical meaning of the escape velocity). Since, —as we will see-the higher orbitals are built by additive particles to the ground state so this ground state should be built by the least amount of the particles. So, Equation (75) neglected a factor coming from the integration which had to be considered. Suppose that the total electric energy between the giant proton $\mathrm{A}, \&$ the giant electron $\mathrm{B}$, is just more than the total binding energy inside $A$, so that $A$, would be evacuated from its charges. We want to estimate the least amount of the charges which cannot do evacuation such that adding any other amount of the charge-even one charge-would lead to increase the electric potential more than the binding energy so evacuation begins to take place. Since that, we want to prevent addition of extra proton. Therefore, the correct estimation needs to suppose that the protons on the surface of A, are pulled to B, one by one by the least possible power. Since each of the two spheres has curvature then there is a point $O$, on the surface of $A$, is the nearest point to $\mathrm{B}$. The electric work done (by B) $w=F \int \mathrm{d} x$ in this point is in its minimum form. Since that, we can see sequent operations on this point. In each operation a single proton would move from the point $\mathrm{O}$ and pulled towards $B$, where when be added to the electrons of $B$, aborts the electric action of one electron. So the electric potential between A \& B would decrease in the second operation by the term one while the binding energy inside $A$, could be considered constant. That is because $n \gg p$ which means that the proton when be 
lost from A, leads to neither considerable loss in the mass nor decrease in the radius of $\mathrm{A}$. The total operations would appear finally as;

$$
G \frac{m(n)}{r}\left(m \frac{n}{p}\right) \int \mathrm{d} p=k \frac{e^{2}}{R} p \int_{0}^{p} \mathrm{~d} p \rightarrow G \frac{m^{2} n^{2}}{r}=-\frac{1}{2} k \frac{e^{2} p^{2}}{R}
$$

where, the huge mass $\left(m \frac{n}{p}\right)=$ constat $\&$ the negative sign $=$ opposit action.

The least amount of the binding energy which is required to prevent evacuation and to enable the sphere to orbit is formulated from (74) \& (75) as;

$$
\therefore G \frac{m^{2} n^{2}}{r}=\frac{1}{4} k \frac{e^{2} p^{2}}{R}
$$

You can see that loss of-even-all the protons in the mind of the left side means loss of mass $=10^{-12}$ of the total mass (so it is constant), while that in the right side means $p-p=0$ (mathematics should think sometimes as a physician). Briefly, the estimated minimal energy - to avoid an extra proton-should be done by integral process as one by one. The above 4 subchapters are necessary to the next chapter.

\section{The Giant Atom Orbit}

We showed that the giant proton to rotate-by the electric field-in its orbit as one body (or as huge charges but not as individual protons) needs internal gravitational binding energy (the particles by its own gravity bind with each other). From (74) \& (76), the least required binding energy has to be in the form

$$
G \frac{m \cdot n}{r}\left(\frac{n}{p} \cdot m\right)=k e^{2} p / 4 R=\left(\frac{n}{p} \cdot m\right) \frac{v^{4}}{4}
$$

where; $r=r_{c} n^{1 / 3}$ is the radius of the sphere of the giant proton, $R$ is the radius of the orbit and $(m \cdot n)$ is the entire mass of the giant proton while $(m n \div p)$ is the mass of the huge proton. (From now we will use $n$ to stand for neutrons number of the giant proton). This equation could be considered as the cardinal equation of the orbit from which we can conclude a direct relation between the number of the neutrons $n$ of the giant proton and the average speed $v$ of the giant proton throughout its orbit radius $R$. So this relation takes the form of:

$$
v^{2}=4 G m n^{2 / 3} / r_{c}
$$

where: $r_{c}$ is the spacing between particles inside the sphere. Or could be written as

$$
v^{3}=n\left(4 G m / r_{c}\right)^{3 / 2}
$$

Now, let us re-go to the giant proton unit which needs the ratio $\frac{n}{p}=1.2123 \times 10^{12}$.

From Equation $(70), \quad v \approx 5.07 \times 10^{4} \mathrm{~m} / \mathrm{s}$ this is the velocity of the giant atom orbit in the ground state. It is very near to the astronomic velocity of the mercury planet (the nearest to the sun) which has mean value of astronomic orbital 
velocity $v=4.74 \times 10^{4} \mathrm{~m} / \mathrm{s}$ with error factor in our estimation about 1/1.07.

Since it is known that our sun losses gradually some of matter in production of heat. It is known that it lost about seven percent of its natal mass [11] so if the present mass of the sun $=1.99 \times 10^{30} \mathrm{~kg} \&$ if its natal mass was, $A$

$$
A(1-0.07)=1.99 \times 10^{30} \rightarrow A=2.13 \times 10^{30} \mathrm{~kg}
$$

By the inverse square law, we have -also-natal orbit speed;

$$
v=1.07 \times 4.74 \times 10^{4}=5.07 \times 10^{4} \mathrm{~m} / \mathrm{s}
$$

$\therefore$ The orbital velocity of the ground state of the giant atom is completely fixed on the natal orbital velocity of the mercury planet $=5.07 \times 10^{4} \mathrm{~m} / \mathrm{s}$.

This is the circular giant atom orbit, but the astronomic orbit of the mercury planet is elliptical with eccentricity $e=\frac{R_{p}}{R}=\left(\frac{v_{x}}{v}\right)^{2}=1.54$ [12] [13].

Equation (78) tells us that the orbiting giant proton possess number of particles just enough to overcome the electric attractive energy. If the orbit was elliptical then this energy would be maximal at the perihelion $R_{p}$ therefore this quantity of the particles has to be enough to overcome this maximal energy. This means that we have to substitute in (78) at the perihelion (minimal distance). From (77) we can conclude that $n^{2}$ is proportional with $\frac{R_{p}}{R}$ therefore we have to multiply $n$ by $\sqrt{1.54}=1.245$.

This is a constant factor should not be introduced in any other mathematic operation. It is a factor just to guarantee that the quantity $n$ is ready to overcome the maximal possible electric energy and to orbit as one body. Putting the value of $r_{c}$ and putting the value of the average speed $v$ and by substitution in equation 78 which appears now as;

$$
1.245 v^{3}=n\left(4 G \frac{m}{r_{c}}\right)^{3 / 2}
$$

So, we can get the number of the neutrons $n$ of the orbiting giant proton;

$$
n=1.245\left(2.44 \times 10^{45}\right)=3.009 \times 10^{45}
$$

This is the least possible quantity of the particles in the orbiting giant proton whose binding energy can overcome the total electric energy at the nearest point (perihelion). From the first equality of Equation (77) we can conclude that;

$$
p^{\frac{1}{3}}=\left(\frac{n}{p}\right)^{\frac{5}{3}} \frac{R}{A}
$$

where, $A$ is a known constant.

From the second equality of the same equation we can reformulate:

$$
p=m(n / p)(v)^{2} R \div k p e^{2}
$$

By dividing over (82) 


$$
\therefore p^{\frac{2}{3}}=B\left(\frac{p}{n}\right)^{\frac{2}{3}}
$$

where, $B$ is another known constant.

$$
\therefore p=2.482 \times 10^{33}
$$

Or, we can conclude the value of $p$ directly as

$$
\begin{gathered}
p=n(p / n)=3.009 \times 10^{45} / 1.2123 \times 10^{12}=2.482 \times 10^{33} \\
\therefore p^{2} / n=2.047 \times 10^{21}
\end{gathered}
$$

By substitution in Equation (73) (or from the above equations)

$$
R=5.41 \times 10^{10} \mathrm{~m}
$$

since, the mean value of the orbital radius of the nearest planet (mercury) equals about $5.791 \times 10^{10} \mathrm{~m}$ so we have error factor about 1.07 or about the inverse of the error factor of the orbital speed. Since the sun natal mass $=2.13 \times 10^{30} \mathrm{~kg}$ (85).

From (84) and by using the inverse square law we can estimate the real natal orbital radius:

$$
R=(5.791 \div 1.07) \times 10^{10}=5.41 \times 10^{10} \mathrm{~m}
$$

$\therefore$ The estimated velocity \& radius of the orbit of the giant atom in its ground state is fixed well on the astronomic estimated natal velocity \& radius of the mercury orbit.

Later on-in a separate discussion-we would see that each a planet was formed of number of these giant charges then in a next stage and after beta decay and formation of atoms the inter-particles distances would increase and consequently the giant charge radius would increase which would lead to inability of the unit to keep its charges which would lead to escape of the charges and consequently attraction and unification of the units to form the planet where Newton's laws would receive the file of the motion as the same $R \& V$ and converse it without modification by the same square inverse law in its gravitational form. Unification is not a simple classic addition but it is quantum overlapping of the waves to maintain the same radius \&speed. The law is the additive energy (gravitational plus the electric) is constant. This stability is controlled by the count of the additive spheres. As example if in the initial condition, the gravitational energy was about zero while the electric energy was hundred joule, and we have one giant proton in the orbit, then if this giant proton lost half of its charges then fifty percent (of the final condition) of the units would collect to maintain this initial condition and so on.

\section{The Higher Giant State}

The giant atom in its ground state is the smallest inversed giant atom whose giant proton can orbit its giant electron. The inversed atom is the mathematic 
basis of the giant atom. The created (or the unit) giant charge is the physical unit of the giant charge while the huge charge is the physical basis of the giant orbit. So the ground state is formed of $(p)$ of the huge wave length $\left(\lambda_{u}\right)$ or $p^{2} / n$ times $\lambda$

$$
\left(p^{2} / n\right) \lambda=\left(\lambda_{u}\right) p=2 \pi R
$$

The higher levels (the complicated giant charge which represent the planets next to mercury planet) could be constructed by the same above basis. We suggest (recalling Equation (54)) that; the higher states of the complicated giant proton have number of protons $=p \cdot d$. Where $d$-in this chapter-means multiplication of $(p)$ of the giant proton by a factor lies between 1 and 10 and so it obeys the relation:

$$
1<d<10
$$

Such that, the product $p \cdot d$ means protons of the giant proton times $d$. We suggest for the complicated giant electron the factor $D$, where $D$-in this chapter-is a factor obeys the same relation as

$$
1<D<10
$$

Such that $D$ means electrons evacuated giant electron (giant neutron).

From above we can estimate the ratio between mass of the complicated giant electron to that of the complicated giant proton $=1844$.

$$
M_{e} \div M_{p}=1844
$$

In the giant atom (the ground state) $p=p^{\prime}$ where $p$ and $p^{\prime}$ are the number of the protons in the giant proton and that of electrons in the giant electron respectively.

In the complicated atom, the system allows that:

The number of the protons in the complicated giant proton $=p_{1}$ while the electrons of the complicated giant electron $=p_{2}$ such that:

$$
\begin{aligned}
& p_{1}=p d \\
& p_{2}=p=p^{\prime}
\end{aligned}
$$

\section{The equations packet of the higher states:}

The motion of the giant proton in a higher state is controlled by;

\section{As a huge proton:}

Its angular momentum obeys;

$$
[\lambda(p / n) d](v / d)(m \cdot n / p)=l \hbar
$$

where $\lambda=l \cdot \lambda / l=\lambda=$ Bohr wave length and $l$ is the level number $=43$.

The electric force gives

$$
(m \cdot n / p)(v / d)^{2}=k e^{2} p^{\prime} \div R \cdot d^{2}
$$

where $p^{\prime}=$ number of electrons in the giant electron $=p$.

We can notice that the mass of the huge proton in the complicated giant proton still equals; $m \cdot n / p$.

\section{As a giant proton:}


The electric force gives

$$
[(m \cdot n) d](v / d)^{2}=k \frac{p d}{R d^{2}} e^{2} \cdot p^{\prime}
$$

where the big bracket is its mass, $\left(e \cdot p^{\prime}\right)$ is the number of the electrons in the complicated giant electron while $(e \cdot p \cdot d)$ is the number of the protons in the complicated giant proton.

\section{The End of Compton State and Reality of the Particles Number}

The matter density in condensate like nucleus and neutron star is about $5 \times 10^{14}$ $\mathrm{g} / \mathrm{cc}$ (with particle spacing about $2 \times 10^{-15} \mathrm{~m}$ ). In such high density $\beta$ decay is forbidden because decay result in excess particles leading to decrease spacing between particles but neutron degenerate refuse-by Pauli's exclusion-this decrease [14] [15]. Our condensate model differs from neutron star and consequently needs deep insight. First, we don't now search for how such high density was formed. Let us begin-at least here-from the point of; if such sphere had exist (perhaps in early universe) then the study would only search in physics of such sphere. In the giant charge unit neutron decay is not forbidden but it has no chance. No chance because the time need for GCU to grow into Gc equal $d t=t_{o}=0 \quad$ (as we say that the moving body has mass more than the rest one where this excess mass needs no time). Since, - in the alternative relativistic solution-the rest length equals the relativistic length so, the zero time in this solution is really zero time where no time dilatation. But $\beta$ decay is exponential function needs time $\Delta t . \beta$ decay is not forbidden in GC because it would (unlike neutron star) not lead to decrease spacing. Although the density of matter is very high yet the net binding energy is very weak As we showed all energies were withdrawn and transformed into particles except that bit residual energy. When $\beta$ decay produces excess particles which of course need to occupy space then instead of increase of the density there would be other solution suitable for the giant atom which is escape of the charges. Now, $\beta$ decay inside the giant charge means production of excess protons which would do repulsion with the initial protons. Production of excess charges would lead to that the left side of the inequality form of Equation (74) would lead to that, the protons of the giant proton (and the electrons of the giant electron) would be attracted together as singled particles. This would result in evacuation of the sphere from the charges. After that, excess $\beta$ decay would lead to formation of the atoms and consequently much increase of the inter-particles distances giving increase in the radius of the giant charge and consequently decrease in the gravitational binding energy inside the giant charge which would result in escape of any residual charges of the giant orbit (you have to go back to Equation (75) in its inequality form) and so it appears as evacuated giant sphere (neutral body formed of atoms). This would results in attraction of these neutral bodies by the effective gravitational 
field and so allowing the identical orbitals (having the same number of waves and the same wavelength - as in Equation (67) —of the same planet) to interfere and form finally the solar system. The classic dynamics would receive the file of the orbital motion to preserve it without modification. This means that the emergent deficit in the initial condition (electric giant atom) is treated urgently and definitely by overlapping the waves of the identical orbitals of the units of the same planet (and of the sun units). This means that the total energy $\left(E_{g}+E_{e}\right)$ is conserved by the interference of the identical orbitals (which have the same wavelength). The initial condition at the giant charge appeared as; $E_{e}+\sim 0=$ the final condition (after union of the units of the same planet and union of the units of sun) $=0+E_{g}$.

From the equality mentioned in Equation (21), the giant electron needs number of particles 1840 times as massive as the giant proton, hence, the latter orbits around the first. Hence, we can define the orbit length $R_{S}$ and the orbital speed $v_{S}$

In the stage of the neutron decay the familiar atoms are formed, consequently, the next results would be established:

1) $r_{c}$ and $v$ inside the sphere $S$ would vanish and the free charges escape from the sphere.

2) The electrostatic potential between the giant electron and its giant proton vanish.

3) The Newtonian gravitational potential would predominantly receive the file of the orbital motion to conserve $R_{S} \& v_{S}$.

From 1, we can conclude the next results:

The relativistic number of the particles would vanish so, we return to $n=n_{o}$ and $S=s$.

The Newtonian gravitational potential of the solar system orbitals needs union of enough number of $s$ (not $S$ ) to conserve $R_{S} \& v_{S}$.

From all above, we conclude that we return again to the common physics which states that the charges quantity is conserved (generally, the sum of the baryon or lepton number of all incoming particles is the same as the sum of the baryon numbers of all particles resulting from the operation): we began with number of charges inside the sphere $s$ equal $p$ and then it became relativistic $P=\gamma^{2} p$ (and also $n=\gamma^{2} n_{o}$ ) then returned again into its original nonrelativistic form $p$ (and also $n$ ) but it escaped outside the sphere. That is to say: particles cannot be created or destroyed except in pairs, where one is ordinary and the other is an antiparticle.

\section{The Solar System}

We know that the solar system is formed of nine planets rotate around the sun. The nearest one to the sun is mercury which has orbit radius $=5.8 \times 10^{10} \mathrm{~m}$ and orbital speed $v=4.74 \times 10^{4} \mathrm{~m} / \mathrm{s}$. The following table shows the relative parameters which the planets obey [12] [13] [16] [17] 


\begin{tabular}{ccccc}
\hline & Mercury & Venus & Saturn & Pluto \\
\hline $\mathrm{v}$ & $1\left(=4.74 \times 10^{4} \mathrm{~m} / \mathrm{s}\right)$ & $1 / 1.36$ & $1 / 5$ & $1 / 10$ \\
$\mathrm{R}$ & $1\left(=5.79 \times 10^{10} \mathrm{~m}\right)$ & $(1.36)^{2}$ & 25 & 100 \\
\hline
\end{tabular}

Our suggestion states that; each planet had formed (historically) of similar and homologous units. The number of the planet units could be concluded by dividing the planet mass over the mass of the giant proton. Each of these units had behaved as a giant proton. Each planet unit had a sun which had behaved as a giant electron.

The planet unit had rotated-on a base of quantum mechanics-around its sun unit forming a system of "giant atom".

The old system had finished by union of all the sun units to form one "sun" and union of the identical planet units to form one planet (interference of the identical orbitals). So the different planet units had formed nine planets. So-now-we have one sun and nine planets. We also suggested for the solar system the following:

The units of the same planet had the same $R \& v$ therefore after beta decay they pulled to each other and collect in one body mass. Also do the electrons evacuated giant electrons. So we would have one planet orbits its special sun. The units of another higher state have another $v$ and $R$ therefore they collect in a higher level with radius of orbit $=R \cdot d^{2}$.

Because of the radii of the orbits of the units of the different suns are not so away from each other comparative with the units of the different planets so they collect in one mass that is; one sun and nine planets. Mercury orbit represented the ground state of the solar system motion. The higher planets obey; $R^{\prime}=R \cdot d^{2} \rightarrow v^{\prime}=v / d$.

\section{The Ratio " $M_{s} \div \Sigma M_{p}$ "}

It is the ratio between mass of the sun and the summed masses of all planets.

Our estimation gave; $M_{s} \div \Sigma M_{p}=1840$

The astronomic ratio $\approx 600$ where the sun natal mass $=2.13 \times 10^{30} \mathrm{~kg}[11]$

Therefore, there is error in our estimation $=1840 \div 600=3$

So we suggest for dealing this error that; there were 3 orbiting giant protons for each giant electron as; $(\mathrm{O}, \mathrm{y}, \mathrm{z}),(\mathrm{x}, \mathrm{O}, \mathrm{z}) \&(\mathrm{x}, \mathrm{y}, \mathrm{O})$.

$$
\therefore M_{s} \div \Sigma M_{p}=1840 \div 3=600 \text { times }
$$

Three orbiting giant protons for each giant electron should make elliptical orbit (even in the ground state) and give spatial shape as if it was real giant atom.

Union of the three orbits (overlap of their waves) in the Newtonian stage may explain the final shape of the orbit.

\section{The Giant Charge Interior}

After we knew physics of Compton sphere and how it replicated till it becomes 
giant charge then this giant charge is the final macroscopic product. Its interior would forget the entire microscopic events and would remember only: the huge charge (which is formed of one charge and $\frac{n}{p} \approx 1.2123 \times 10^{12}$ neutrons) is the unit of GCU. Then, the mass of the huge charge $=m_{u}=m \frac{n}{p} \& u_{g u}$ is the gravitational energy per a huge charge so the huge charge is the one which describes this wavy behavior. The huge proton has a classic radius $r_{u}=3 \times 10^{-16} \times \sqrt[3]{10^{12}} \approx 3 \times 10^{-12} \mathrm{~m}$ but its wave length is shorter. So, we can consider that one proton with $\frac{n}{p}=1.2123 \times 10^{12}$ neutrons is encapsulated by one huge wave hence the momentum $p$ appears as expression of this wave as: $p=\sqrt{2 m_{u} E}$.

Anyway, each particle of the giant charge (the adult) appears with rest mass = $m_{s}$ and with zero energy except its $u_{g}$ which appears as the residual binding energy of the system. So it has: $u_{g u}=\frac{3}{5} G \frac{m \cdot n\left(m \frac{n}{p}\right)}{r}$ where the product between the brackets is the mass of the huge charge (notice that, $=m_{s}$ ). The ratio $\frac{n}{p}$ and the density of matter and consequently the density of energy inside the sphere are conserved. Equations (23) \& (24) describe the density of energy of the particle inside the Compton's sphere and also inside the giant charge. This form of the two equations guarantees that $v$ never exceeds $c$, therefore the entire energies for a proton \& a neutron inside the giant charge appear as

$$
\left(u_{e}+u_{G}\right)+\left(u_{c}+u_{G}\right)+u_{g u} \equiv\left(u_{e}+u_{m}\right)+\left(u_{c}+u_{m}\right)+u_{g u}=m c^{2}
$$

where: $u_{m}$ is the magnetic energy subdivision. The motion inside the giant sphere is withdrawn and appears here, in the macroscopic version as rest energy except a bit of energy is still alive and represents the net residual energy of the system. The particles by this residual energy possess matter waves. We can estimate the wave length $\lambda \approx 10^{-24} \mathrm{~m}$ therefore this estimated wave length is comparatively very small compared with the spacing $r_{c}$ which means deviation towards the classic mechanics. We discussed all the energetic terms inside our sphere. Because the pair term of this sphere depends on the spin coupling interaction of its nucleons [17] \& it is odd or even number (as in the liquid drop model of the nucleus) and because the pairing term decreases with the even mass number $A$ and equals zero if it was odd [18] and because $A$ of the Compton's sphere is very great so we can consider the energy of this term neglected if compared with $u_{G}$. Any way, we can consider the mass number is odd so the term equals zero. We have also to remember that all the equations of the Compton's sphere are constrained and directed mathematically to realize the initial conditions.

\section{Diagrammatic Summary}


1) $k \frac{e^{2}}{r_{o}}=E_{o}=m_{o} c^{2}$

2) The intuit; $k \frac{e^{2}}{r_{o}} \times 1=E_{o}=m_{o} c^{2} \rightarrow$ macroscopic state of the Compton sphere in a rest state $\rightarrow m=m_{o} \& r=r_{o}$ therefore the energy density of a particle defined by Equation (1) is still describing the energy density of the giant particle (where $v$ never exceed $c$ ).

3) Any other additional solution should be constrained by this intuit. This equation carries three results. The first is; the squared $c$ does not mean real speed, so the particle is in a rest state without relativistic relations. The second is that; we succeeded to abort-physically-the repulsive electric energy. The third result appears if we wrote $c$ in the next relativistic form;

$$
k \frac{e^{2}}{r_{o}}\left\{\left(1-\frac{1}{\gamma^{2}}\right)+\frac{1}{\gamma^{2}}\right\}=E_{o}=m_{o} c^{2}=m_{o}\left[\left(c^{2}-\frac{c^{2}}{\gamma^{2}}\right)+\left(\frac{c^{2}}{\gamma^{2}}\right)\right]=m_{o} c^{2} \downarrow
$$

$(\downarrow)(\downarrow)$

(Original relativity $\left.=\gamma^{2} m_{o}^{2}=m^{2} \leftarrow v_{1}^{2}\right)+\left(v_{2}^{2}=2 \frac{u_{\mathrm{g}}}{m}\right)$

$\downarrow$

Giant Charge Unit $=$ Compton Sphere $\leftarrow\left(v^{2} \equiv c^{2}\right) \rightarrow m_{o} c^{2} \uparrow$

4) On the same way, Equations (31)-(38) can abort the exclusive quantum energy due to the spacing between particles of the sphere.

The original relativity should be constrained by the above intuit $\rightarrow$ alternative relativistic solution; $m=m_{o}, r=r_{o}, \quad v \equiv c \& n=\gamma^{2} n_{o}=10^{24} n_{o} \rightarrow$ Giant Charge $=$ relativistic compton sphere $\rightarrow$ the solar system.

\section{Larmor's equation problem:}

It is clear that the circular (or the elliptical) orbit of the giant atom differs from the normal atom in which the electron is described by the wave function where it appears as cloud spread all over its standing wave (not orbiting in a circle otherwise it would radiate till finally fall in the nucleus). If we go to the mathematic derivation of Larmor's equation and as approximation comparison with orbit of a hydrogen atom: if the number of the elementary charges in the giant atom $=q$ and its orbit radius $=R$, and if $A$ is a constant, and using J.J Thomson treatment with the suitable modification

$\therefore \quad$ The energy lost $P$ per an elementary charge per second, $=A q a^{2}=A q\left(v^{2} / R\right)^{2}$. This gives ratio between $P$ of the giant proton and that of the electron of a hydrogen atom as; $P_{g} / P_{e} \approx 10^{33} \times 10^{-7} \times 10^{-42} \approx 10^{-16}$. This means that each proton of the giant proton can orbit in a circle for a long time without emitting considerable radiation. From another side, it is quite clear that inside the giant charge itself and according to the equality-which we showed in the beginning of this study-between $E_{e}$ and $E_{G}$ there would be neither acceleration nor emitting radiation. Finally you notice that the giant atom physics can carry all the information's about the system like radius and speed of the orbits, 
the $\left(M_{s} \div \Sigma M_{p}\right)$ ratio, the elliptical shape and its eccentricity, and also degree of the orbit decline and spin of the planets.

\section{Results:}

1) The density of the giant charge defined by the spacing $r_{c}=3.127 \times 10^{-16} \mathrm{~m}$ is the density of the "rest" macro-state in which the particles condensate so much that they feel neither their charges nor their quantum effect, while the density of the Compton sphere defined by $r_{c}=3.127 \times 10^{-16} \div \sqrt{1-\frac{n}{p x \mu}}$ is the density of the micro-states in which the particles condensate \& replicate by the relativistic alternative solution.

2) If we multiplied the electric subdivision-of the rest energy of the particle-by one, and if we factorized and analyzed this one-as Equations (5) \& (6) and then followed up the relativistic micro-state of Equation (7) we would get the solar system as in Equations (79), (86) \& (95).

3) We succeeded to fix the coulomb's form of the electric energy on the rest energy form as in Equation (3). We also succeeded to do a junction between the classic \& the quantum mechanics.

\section{Conclusion:}

The giant atom like system is a scientific truth. This is confirmed by what we found as complete coincidence between the parameters of such a system and that of the solar system. Such a system carries the scientific seeds of physics of self-replication.

\section{References}

[1] Emad, E. (2017) Physics of the Relativistic Giant Atom. International Journal of Theoretical and Mathematical Physics, 7, 68-89.

[2] Griffiths, D.J. (1995) Introduction to Quantum Mechanics. Prentice-Hall, Upper Saddle River, New Jersey, 155.

[3] Garay, L.J. (1995) Quantum Gravity of Minimum Length. International Journal of Modern Physics, 10, 145. https://doi.org/10.1142/S0217751X95000085

[4] Bailey, D. (2011) Semi Empirical Nuclear Mass Formula: Strings and Binding Energy. PHY357: Strings \& Binding Energy. University of Toronto.

[5] Doolittle, A. (1982) Density of States and Fermi Energy Concept Alzergin and v. Korepin. Letter in Mathematical Physics, 6, 12-18.

[6] Heisenberg, W. (1949) The Physical Principle of Quantum Theory. Dover Publications, Mineola, New York.

[7] Regal, C.A., Greiner, M. and Jin, D.S. (2004) Fermi Energy. Physical Review Letters, 92, Article ID: 040403.

[8] Golub, R. and Chordson, D.R. (1991) Ultracold Neutrons. Adam Hilger, Bristol.

[9] Emad, E. (2015) The Electrostatic Energy in a Rest Form; Evidences and Recommendations. International Research Journal of Pure and Applied Physics, 3, 9-14.

[10] Emad, E. (2009) The Giant Atom Like System. Advanced Studies in Theoretical Physics, 3, 14-15.

[11] Kaufman, W.J. (1979) Planets and Moons. W. H. Freeman, San Francisco. 
[12] Simon, J.L. and Bretagnon, P. (1994) Numerical Expression for Formulae \& Mean Elements for the Moon \& Planets. Astronomy and Astrophysics, 282, 663-683.

[13] Strom, R.G. and Sprague, A. (2003) Exploring Mercury: The Iron Planet. Praxis Public Ltd., Chichester.

[14] Baym, G. (1995) Neutron Stars: Observing the Properties of High-Density Nuclear Matter. Nuclear Physics A, 590, 233. https://doi.org/10.1016/0375-9474(95)00238-V

[15] Dwivedi, V. (2012) Condensates in Neutron Star Interiors. Phys 569: Emergent States of Matter.

http://guava.physics.uiuc.edu/ nigel/courses/569/Essays_Fall2012/Files/dwivedi.pdf

[16] Anderson, J.D., Colombo, G., Esposito, P.B., Lau, E.L., et al. (1987) The Mass Gravity Field and Ephemeris of Mercury. Icarus, 71, 337-349. https://doi.org/10.1016/0019-1035(87)90033-9

[17] Konopliv, A.S., Banerdt, W.B. and Sjogren, W.L. (1999) Venus Gravity: 180th Degree and Order Model. Icarus, 139, 3-18. https://doi.org/10.1006/icar.1999.6086

[18] Krane, K. (1988) Introductory Nuclear Physics. John Wily \& Sons, Hoboken.

[19] Turtur, C.W. (1985) An Explanation of Electron Mass by the Energy of Its Fields. https://www2.ostfalia.de/export/sites/default/de/pws/turtur/FundE/English/electron engl.pdf 


\section{Appendix}

Actually Lorentz postulated that the intrinsic energy of the charged particle is due to its electromagnetic behavior [19]. So let us estimate the part of the electromagnetic energy due to the electric field and add it to that part due to the motion (spin) of the particle (magnetic energy). Now in each infinitesimal volume we have the electric energy density in the form of; $(\Delta E)_{1}=\omega(E \cdot E) / 2$. Where $\omega$ is the permittivity constant of space and $(E \cdot E)$ is the dot product of the electric field vector (the result is scalar). And all over the spherical volume we have the electric potential per particle

$$
u_{e}=\frac{e^{2}}{32 \pi^{2} a^{4} \omega} \int_{0}^{2 \pi} \int_{0}^{\pi} \int_{r}^{\infty} r^{2} \sin \theta \mathrm{d} r \mathrm{~d} \theta d \phi=\frac{e^{2}}{4 \pi \omega(2 a)}=\frac{1}{2} m_{o} c^{2}
$$

where $\omega$ is the permittivity constant. We would estimate the magnetic field $B$ in z-direction (in S.I. units) postulating a literal circular motion of the charged particle with radius of rotation equals its classic radius $a=r$. So we would estimate the magnitude of $B$ in z-direction due to motion of the charged elementary particle in a circle with length $l=2 \pi a$ where $a=r$. From Biot-Savart law in its simple form:

$$
B=\frac{\mu}{4 \pi} i \frac{\int \mathrm{d} l}{a^{2}}=\frac{\mu i}{2 r}
$$

where $\mu$ is the permeability constant of the space and $i$ is the electric current of the charged particle as: $i=\frac{e}{T}=\frac{e v}{2 \pi a} \rightarrow B=\frac{\mu e v}{4 \pi a^{2}}$. The magnetic moment is estimated as: $m=i \pi a^{2}$.

$\therefore$ The energy part due to spin $=u_{s}=-\boldsymbol{m} \times \boldsymbol{B}$ where $\times$ is the cross product which gives in z-direction: $\frac{v^{2}}{4 \pi 2 a} e^{2} \mu=u_{s}$.

Put $v=c$ and put $\omega \mu c^{2}=1$.

$\therefore u_{s}=\frac{e^{2}}{4 \pi \omega(2 a)}$ or, we can estimate it directly from Maxwell equations as:

$$
(\Delta E)_{2}=B^{2} / 2 \mu=E^{2} \mathcal{E} / 2 \quad \text { where } B=-E / c
$$

And the total energy is estimated by adding the two parts of energy

$$
\therefore u=\Delta E=2 \frac{e^{2}}{4 \pi \omega(2 a)}=m_{o} c^{2}
$$

If we applied the above mathematic manipulation in Equation (2) (the giant charge unit) then either in the macroscopic state (rest state) or the microscopic state $(v \approx c)$ the result would be the same

$$
\frac{1}{2} m_{o} c^{2}+\frac{1}{2} m_{o} c^{2}=\frac{1}{2}\left(k \frac{e^{2}}{r_{o}}-\frac{k \frac{e^{2}}{r_{o}} n}{p x \mu}+\frac{k \frac{e^{2}}{r_{o}} n}{p x \mu}\right)+\frac{1}{2}\left(k \frac{e^{2}}{r_{o}}-\frac{k \frac{e^{2}}{r_{o}} n}{p x \mu}+\frac{k \frac{e^{2}}{r_{o}} n}{p x \mu}\right)
$$


where, $\frac{k \frac{e^{2}}{r_{o}} n}{p x \mu}=u_{g}, k \frac{e^{2}}{r_{o}}\left(\frac{r_{o}}{r_{c}} \frac{p^{\frac{2}{3}}}{\left(\frac{n}{p}\right)^{\frac{1}{3}}}\right)=m_{o} c^{2}$. Where, the product inside the brackets $=1$. 